\title{
Maximally Exposed Offsite Individual Location Determination for NESHAPS Compliance
}

by

A. A. Simpkins

Westinghouse Savannah River Company

Savannah River Site

Aiken, South Carolina 29808

This paper was prepared in connection with work done under the above contract number with the U.S. Department of Energy. By acceptance of this paper, the publisher and/or recipient acknowledges the U.S. Government's right to retain a nonexclusive, royalty-free license in and to any copyright covering this paper, along with the right to reproduce and to authorize others to reproduce all or part of the copyrighted paper. 


\section{MAXIMALLY EXPOSED OFFSITE INDIVIDUAL LOCATION DETERMINATION FOR NESHAPS COMPLIANCE}

$1 / 2$

Ali A. Simpkins

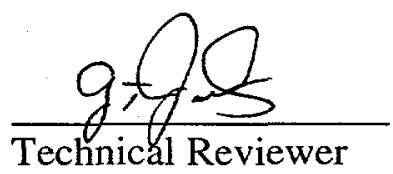

January 2000

Westinghouse Savannah River Company Savannah River Site Aiken, SC 29808
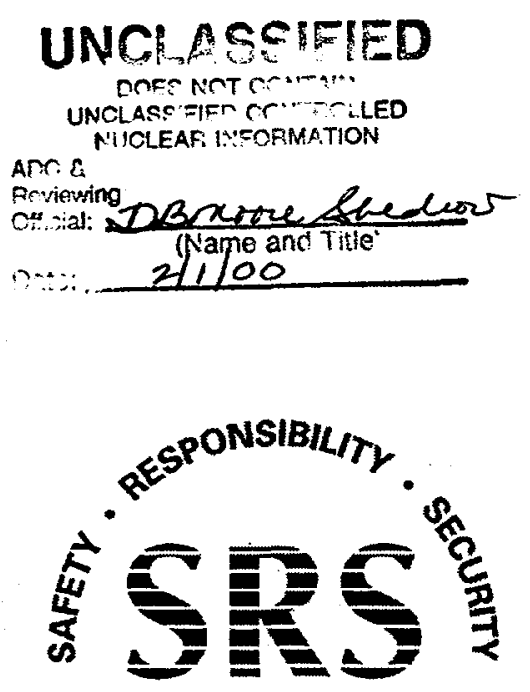

SAVANNAH RIVER SITE 


\section{DISCLAIMER}

This report was prepared as an account of work sponsored by an agency of the United States Government. Neither the United States Government nor any agency thereof, nor any of their employees, makes any warranty, express or implied, or assumes any legal liability or responsibility for the accuracy, completeness, or usefulness of any information, apparatus, product or process disclosed, or represents that its use would not infringe privately owned rights. Reference herein to any specific commercial product, process or service by trade name, trademark, manufacturer, or otherwise does not necessarily constitute or imply its endorsement, recommendation, or favoring by the United States Government or any agency thereof. The views and opinions of authors expressed herein do not necessarily state or reflect those of the United States Government or any agency thereof.

This report has been reproduced directly from the best available copy.

Available for sale to the public, in paper, from: U.S. Department of Commerce, National Technical Information Service, 5285 Port Royal Road, Springfield, VA 22161, phone: (800) 553-6847

fax: (703) 605-6900

email: orders@ntis.fedworld.gov

online ordering: http://www.ntis.gov/ordering.htm

Available electronically at http://www.doe.gov/bridge

Available for a processing fee to U.S. Department of Energy and its contractors, in paper, from: U.S. Department of Energy, Office of Scientific and Technical Information, P.O. Box 62, Oak Ridge, TN 37831-0062, phone: (865) 576-8401

fax: (865) 576-5728

email: reports@adonis.osti.gov 


\section{DISCLAIMER}

Portions of this document may be illegible in electronic image products. Images are produced from the best available original document. 
Key Words Atmospheric Dispersion

Dose Determination

Atmospheric Releases

Retention: Lifetime

\title{
MAXIMALLY EXPOSED OFFSITE INDIVIDUAL LOCATION DETERMINATION FOR NESHAPS COMPLIANCE
}

\author{
A. A. Simpkins
}

Issued: January 2000

$S R T C$

SAVANNAH RIVER TECHNOLOGY CENTER

AIKEN, SC 29808

Westinghouse Savannah River Company

Savannah River Site

Aiken, SC 29808

PREPARED FOR THE U.S. DEPARTMENT OF ENERGY UNDER CONTRACT NO.

DE-AC09-96SR18500 


\section{ABSTRACT}

The Environmental Protection Agency (EPA) requires the use of the computer program CAP88 for demonstrating compliance with the National Emission Standard for Hazardous Air Pollutants (NESHAPS.) One of the inputs required for CAP88 is the location of the maximally exposed individual (MEI) by sector and distance. Distances to the MEI have been determined for 15 different potential release locations at SRS. These locations were compared with previous work and differences were analyzed. Additionally, SREL Conference Center was included as a potential 'offsite' location since in the future it may be used as a dormitory. Worst sectors were then determined based on the distances. 


\section{TABLE OF CONTENTS}

1. INTRODUCTION

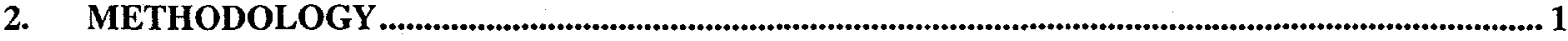

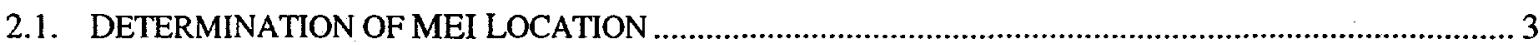

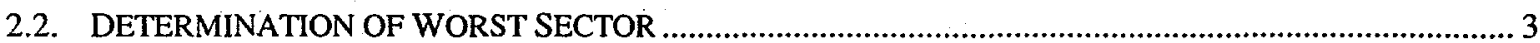

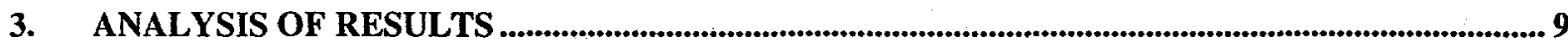

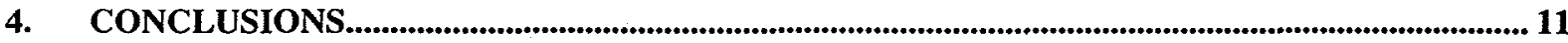

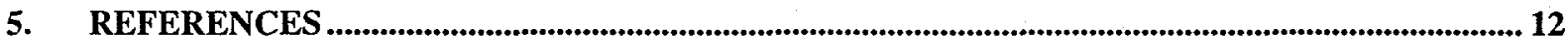




\section{LIST OF TABLES}

TABLE 1. POTENTIAL RELEASE POINTS FOR NESHAPS COMPLIANCE.

1

TABLE 2. DISTANCE TO THE NEAREST RESIDENCE, SCHOOL, BUSINESS, OR FARM FOR

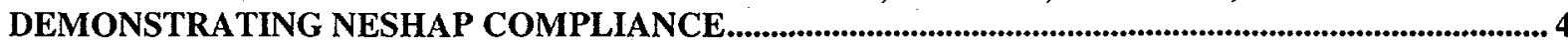

TABLE 3. DISTANCE TO THE NEAREST RESIDENCE, SCHOOL, BUSINESS OR FARM FOR DEMONSTRATING NESHAP COMPLIANCE........................................................................................

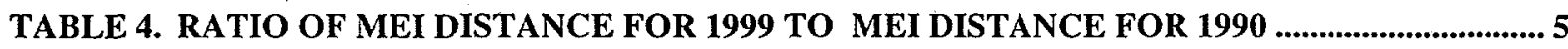

TABLE 5. RELATIVE AIR CONCENTRATIONS AT OFFSITE LOCATIONS CORRESPONDING TO

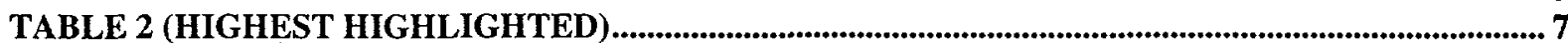

TABLE 6. RELATIVE AIR CONCENTRATIONS AT OFFSITE LOCATIONS CORRESPONDING TO TABLE 3 - (HIGHEST HIGHLIGHTED) ..............................................................................................

TABLE 7. LOCATION OF MEI FOR NESHAPS CALCULATIONS.................................................... 9

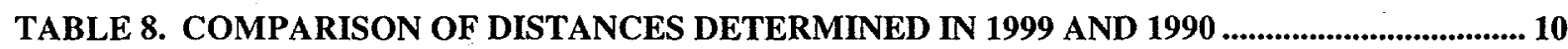

TABLE 9. COMPARISON OF RELATIVE AIR CONCENTRATIONS USING 1999 AND 1990

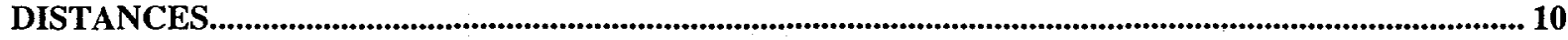

\section{LIST OF FIGURES}

FIGURE 1. LOCATION OF MAJOR SITE AREAS WITH POTENTIAL TO RELEASE

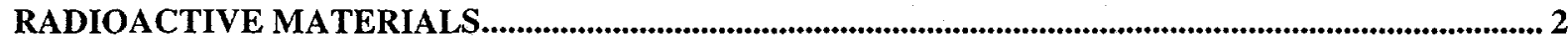




\title{
MAXIMALLY EXPOSED OFFSITE INDIVIDUAL LOCATION DETERMINATION FOR NESHAPS COMPLIANCE
}

\author{
By A. A. Simpkins \\ Westinghouse Savannah River Company \\ Savannah River Site \\ Aiken, SC 29808
}

\section{INTRODUCTION}

The Environmental Protection Agency (EPA) requires the use of the computer code CAP88 (Beres 1990) for demonstrating compliance with the National Emission Standard for Hazardous Air Pollutants (NESHAPS). To demonstrate compliance, the location of the maximally exposed individual (MEI) is needed as input to CAP88. This location is entered as sector and distance to the receptor. These distances and sectors were originally determined in 1990 with virtually no documentation and are determined now with strict documentation.

\section{METHODOLOGY}

To determine the location of the MEI, the nearest offsite individual is identified for each of the sixteen compass point sectors surrounding each potential release point. Next, CAP88 is executed to determine which sector would result in the highest dose (referred to as the worst sector.) A listing of each of the potential release points is shown in Table 1 and is graphically depicted in Figure 1. Table 1 also shows the location of each of the potential release points using the site coordinate system.

Table 1. Potential Release Points for NESHAPS Compliance

\begin{tabular}{|c|c|c|}
\hline Location & Easting Coordinate & Northing Coordinate \\
\hline A-Area & 51860 & 106670 \\
APT & 75000 & 75000 \\
C-Area & 46230 & 67630 \\
Center & 58000 & 62000 \\
D-Area & 20940 & 65280 \\
E-Area & 58000 & 75000 \\
F-Area & 53970 & 78020 \\
H-Area & 63380 & 71900 \\
K-Area & 40740 & 54130 \\
L-Area & 50460 & 45910 \\
M-Area & 50040 & 104830 \\
N-Area & 51000 & 65000 \\
P-Area & 64800 & 43800 \\
S/Z-Area & 64010 & 73750 \\
T-Area & 17500 & 71500 \\
\hline
\end{tabular}


Figure 1. Location of Major Site Areas with Potential to Release Radioactive Materials

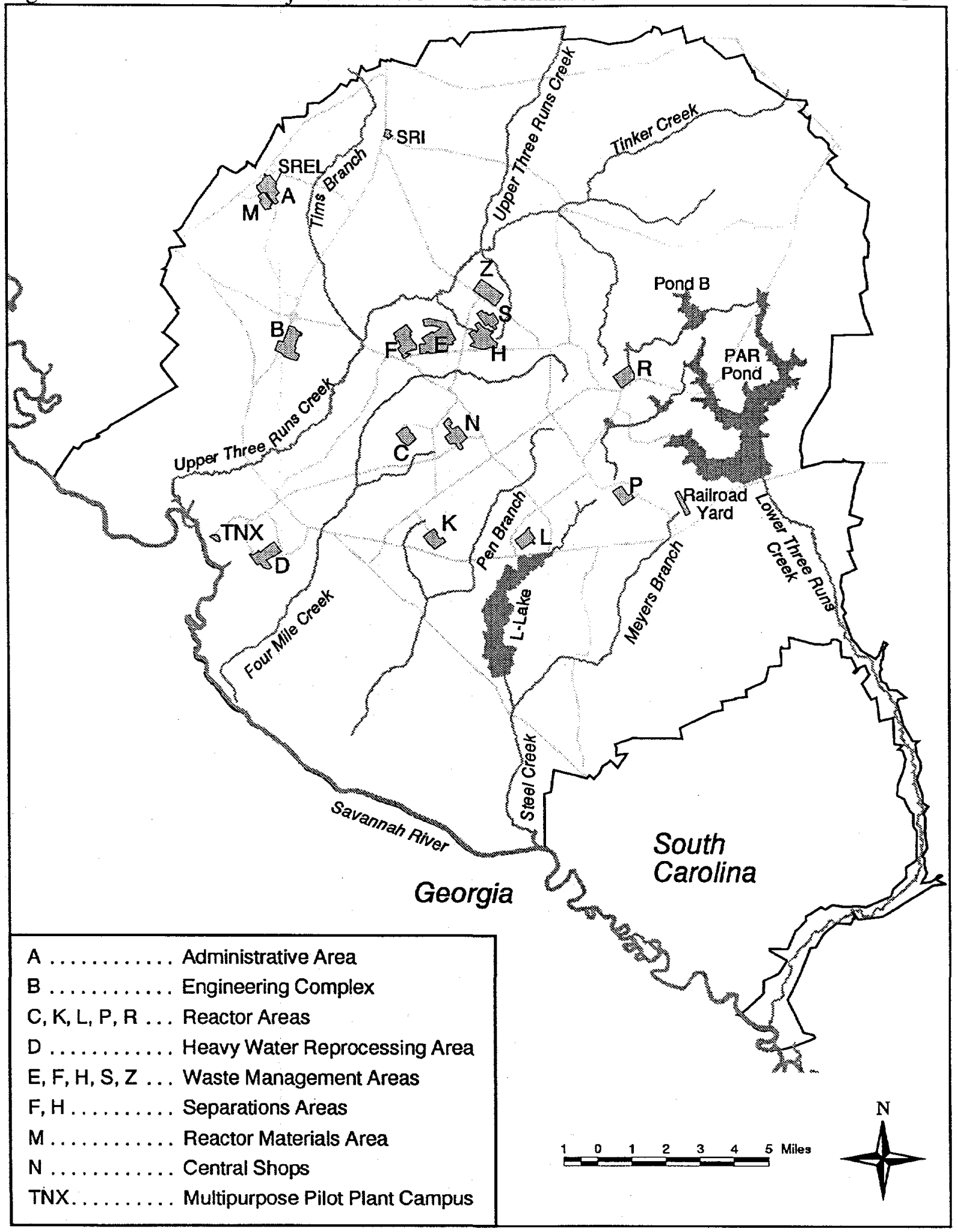




\subsection{Determination of MEI Location}

NESHAPS calculations are performed in accordance with 40CFR61 (EPA 1989). In reference to determining the location of the MEI, Section 92 states:

'Compliance with this standard shall be determined by calculating the highest effective dose equivalent to any member of the public at any offsite point where there is a residence, school, business or office. .....Distances from the points of release to the nearest residence, school, business or office and the nearest farms producing vegetables, milk, and meat.'

The E\&GIS Group was contacted to determine the location of the nearest offsite individual in each of the sixteen compass sectors for each potential release location. Appendix A contains a complete copy of the results of this study (Mackey 1999). Site wide photography taken in 1998 was examined to pinpoint the location of buildings or farms. For conservatism, all cultivated fields were assumed to be vegetable-producing farms.

The Savannah River Ecology Lab (SREL) Conference Center, which is located onsite along Highway 278 , may be used as a dormitory in the future. This location was also considered as a potential residence location. Table 2 shows the distance to the nearest offsite individual for each of the sixteen sectors for each release location and Table 3 contains similar results with the inclusion of the SREL Conference Center. Appendix B shows the distances that were determined in the previous study performed in 1990 .

Table 4 shows the ratio of distances determined in 1999 to those determined in 1990. Looking at Table 4, the only areas that show considerable differences are A Area and D Area, both of which are close to the site boundary. One reason for the differences may be that the photography in 1998 has greater detail. Another reason may be that some of the buildings/farms that were selected using the 1998 photography may not have existed in 1990.

Independent review of the photography for A Area and D Area validated that correct methods were used with the current study. For A Area, the north-northwest sector was looked at closely because this was the location of the worst sector during the previous analysis. To ensure proper selection of nearest building/farm a field verification was preformed for questionable sectors for the A Area release lecation. This field verification determined that there is a habitable structure at the questionable location.

There may be differences when comparing this study to the previous study because of how the sectors were defined. Current methods utilized computer models to overlay exact 22.5 degree sectors centered upon true North. Previous studies might not have used such sophisticated methods to define sectors.

\subsection{Determination of Worst Sector}

Now that distances have been determined as shown in Tables 2 and 3, CAP88 is executed for each of these distances and corresponding sectors to determine which sector would provide the highest dose to the offsite individual. The relative air concentrations, which are directly proportional to dose, are shown in Tables 5 and 6 both without and with considering the 
Table 2. Distance to the Nearest Residence, School, Business, or Farm for Demonstrating NESHAP Compliance

\begin{tabular}{|c|c|c|c|c|c|c|c|c|c|c|c|c|c|c|c|}
\hline \multirow[b]{2}{*}{ Sector } & \multicolumn{15}{|c|}{ Release Area - Distance (m) } \\
\hline & $\mathbf{A}$ & PT & $\mathbf{C}$ & Center & D & E & $\mathbf{F}$ & $\mathbf{H}$ & $\mathbf{K}$ & $\mathbf{L}$ & $\mathbf{M}$ & $\mathbf{N}$ & $\mathbf{P}$ & $\mathrm{S} / \mathrm{Z}$ & $\mathrm{T}$ \\
\hline $\bar{S}$ & 20575 & 20750 & 15102 & 15033 & 7329 & 19235 & 19026 & 18466 & 13401 & 9621 & 19788 & 17874 & 10928 & 19054 & 5 \\
\hline $3 \mathbf{W}$ & 7758 & 23038 & 13477 & 16784 & 5355 & 17637 & 16135 & 19398 & 11102 & 12970 & 7077 & 15119 & 11828 & 95 & 4613 \\
\hline $\mathbf{S}$ & 4761 & 21743 & 12751 & 16203 & 4996 & 16564 & 15328 & 18080 & 10986 & 12513 & 4815 & 14134 & 16535 & 8359 & 4450 \\
\hline SW & 2639 & 15574 & 13236 & 17004 & 5792 & 13316 & 10229 & 15194 & 10957 & 14074 & 3137 & 14786 & 19 & 3893 & 4551 \\
\hline 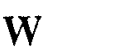 & 2141 & 14625 & 10481 & 13839 & 6390 & 10967 & 9442 & 12817 & 13296 & 16377 & 2440 & 11832 & 14 & 03 & 4816 \\
\hline JW & 1173 & 12396 & 10150 & 90 & 7810 & 11557 & 9996 & 12346 & 12912 & 16446 & 2203 & 11634 & 19459 & 11259 & 5670 \\
\hline 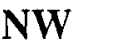 & 699 & 10193 & 11904 & 14434 & 8180 & 10525 & 9450 & 11820 & 13212 & 17200 & 1538 & 13389 & 20252 & 027 & 6221 \\
\hline & 973 & 9932 & 12693 & 15032 & 8291 & 11007 & 9948 & 11623 & 17025 & 19539 & 1342 & 13723 & 19772 & 828 & 6394 \\
\hline$N$ & 1159 & 9478 & 14475 & 15813 & 14868 & 11478 & 10933 & 12380 & 19027 & 21069 & 1932 & 15188 & 19021 & 11795 & 9117 \\
\hline $\mathbf{E}$ & 2626 & 9580 & 17761 & 15987 & 21224 & 13534 & 14180 & 12723 & 21491 & 21372 & 3390 & 16996 & 16182 & 12225 & 8430 \\
\hline & 3770 & 11461 & 20048 & 17228 & 27066 & 15598 & 16290 & 14682 & 22663 & 17868 & 5200 & 19334 & 13493 & 19 & 25253 \\
\hline ENE & 13725 & 12540 & 20209 & 16283 & 23297 & 17575 & 18973 & 15792 & 16431 & 12843 & 14176 & 18584 & 8468 & 15703 & 28951 \\
\hline $\mathrm{E}$ & 21107 & 13408 & 16904 & 13012 & 21689 & 17761 & 19279 & 15872 & 17341 & 13483 & 22659 & 15260 & 9662 & 15974 & 25043 \\
\hline & 24872 & 13106 & 17939 & 15233 & 16802 & 15806 & 17303 & 14046 & 13847 & 11386 & 24661 & 16644 & 9845 & 14389 & 18494 \\
\hline & 28548 & 16035 & 16414 & 15131 & 15032 & 19002 & 19820 & 17815 & 12227 & 10054 & 27997 & 15855 & 9268 & 18351 & 16808 \\
\hline SSE & 27453 & 18700 & 15450 & 15025 & 10533 & 18650 & 19115 & 18078 & 11098 & 9906 & 26800 & 15059 & 9617 & 18656 & 10478 \\
\hline
\end{tabular}


Table 3. Distance to the Nearest Residence, School, Business or Farm for Demonstrating NESHAP Compliance (SREL Conference Center Included - Italics show distances that change as a result of Conference Center Included)

\begin{tabular}{|l|r|r|r|r|r|r|r|r|r|r|r|r|r|r|r|}
\hline \multirow{2}{*}{ Sector } & \multicolumn{1}{|c|}{ A } & \multicolumn{1}{|c|}{ APT } & \multicolumn{1}{|c|}{ C } & Center & \multicolumn{1}{|c|}{ D } & \multicolumn{1}{|c|}{ E } & \multicolumn{1}{|c|}{ F } & \multicolumn{1}{c|}{ H } & \multicolumn{1}{|c|}{ K } & L & M & N & P & S/Z & T \\
\hline S & 20575 & 20750 & 15102 & 15033 & 7329 & 19235 & 19026 & 18466 & 13401 & 9621 & 19788 & 17874 & 10928 & 19054 & 5628 \\
SSW & 7758 & 23038 & 13477 & 16784 & 5355 & 17637 & 16135 & 19398 & 11102 & 12970 & 7077 & 15119 & 11828 & 19795 & 4613 \\
SW & 4761 & 21743 & 12751 & 16203 & 4996 & 16564 & 15328 & 18080 & 10986 & 12513 & 4815 & 14134 & 16535 & 18359 & 4450 \\
WSW & 2639 & 15574 & 13236 & 17004 & 5792 & 13316 & 10229 & 15194 & 10957 & 14074 & 3137 & 14786 & 18419 & 13893 & 4551 \\
W & 2141 & 14625 & 10481 & 13839 & 6390 & 10967 & 9442 & 12817 & 13296 & 16377 & 2440 & 11832 & 20514 & 12603 & 4816 \\
WNW & 1173 & 12396 & 10150 & 13690 & 7810 & 11557 & 9996 & 12346 & 12912 & 16446 & 2203 & 11634 & 19459 & 11259 & 5670 \\
NW & 699 & 10193 & 11904 & 14434 & 8180 & 10525 & 9450 & 11820 & 13212 & 17200 & 1538 & 13389 & 20252 & 11027 & 6221 \\
NNW & 973 & 9932 & 12693 & 15032 & 8291 & 11007 & 9948 & 11623 & 17025 & 19539 & 1342 & 13723 & 19772 & 11828 & 6394 \\
N & 1159 & 8370 & 14475 & 14740 & 14868 & 11478 & 10933 & 12380 & 19027 & 19960 & 1932 & 15188 & 18150 & 10940 & 9117 \\
NNE & 2626 & 9580 & 16390 & 15987 & 21224 & 12160 & 12750 & 11460 & 20210 & 21372 & 3390 & 15690 & 16182 & 12225 & 18430 \\
NE & 3770 & 11461 & 20048 & 17228 & 27066 & 15598 & 16290 & 14682 & 22663 & 17868 & 5200 & 19334 & 13493 & 14319 & 23770 \\
ENE & 12320 & 12540 & 20209 & 16283 & 23297 & 17575 & 18973 & 15792 & 16431 & 12843 & 12740 & 18584 & 8468 & 15703 & 28951 \\
E & 21107 & 13408 & 16904 & 13012 & 21689 & 17761 & 19279 & 15872 & 17341 & 13483 & 22659 & 15260 & 9662 & 15974 & 25043 \\
ESE & 24872 & 13106 & 17939 & 15233 & 16802 & 15806 & 17303 & 14046 & 13847 & 11386 & 24661 & 16644 & 9845 & 14389 & 18494 \\
SE & 28548 & 16035 & 16414 & 15131 & 15032 & 19002 & 19820 & 17815 & 12227 & 10054 & 27997 & 15855 & 9268 & 18351 & 16808 \\
SSE & 27453 & 18700 & 15450 & 15025 & 10533 & 18650 & 19115 & 18078 & 11098 & 9906 & 26800 & 15059 & 9617 & 18656 & 10478 \\
\hline
\end{tabular}

* No Change - Distance to SREL Conference Center greater than distance to the nearest offsite individual so original distance used. 
Table 4. Ratio of MEI distance for 1999 to MEI distance for 1990

\begin{tabular}{|c|c|c|c|c|c|c|c|c|c|c|c|c|c|c|}
\hline Sector & A & APT & C & Center & D & E & F & H & K & L & M & P & S/Z & T \\
\hline S & 1.0 & 1.0 & 0.9 & 1.0 & 0.7 & 0.9 & 1.0 & 1.0 & 1.0 & 1.0 & 1.0 & 1.0 & 0.9 & 0.9 \\
SSW & 1.1 & 1.0 & 0.9 & 1.0 & 0.9 & 1.0 & 1.0 & 1.0 & 1.0 & 0.9 & 1.0 & 1.0 & 0.9 & 0.7 \\
SW & 0.9 & 1.0 & 1.0 & 1.0 & 0.7 & 1.0 & 1.0 & 1.0 & 0.9 & 0.9 & 0.9 & 0.9 & 0.9 & 0.9 \\
WSW & 0.8 & 1.0 & 1.0 & 1.0 & 0.9 & 1.0 & 0.9 & 1.0 & 1.0 & 1.0 & 0.9 & 1.0 & 1.0 & 1.1 \\
W & 0.8 & 1.0 & 0.9 & 1.0 & 1.0 & 1.0 & 1.0 & 1.0 & 1.0 & 1.0 & 1.0 & 1.0 & 1.0 & 0.8 \\
WNW & 0.5 & 1.0 & 1.0 & 1.0 & 0.9 & 1.0 & 1.0 & 1.0 & 1.0 & 1.0 & 1.0 & 1.0 & 1.0 & 1.0 \\
NW & 0.4 & 1.0 & 1.0 & 1.0 & 1.0 & 1.0 & 1.0 & 1.0 & 1.0 & 0.9 & 1.0 & 1.0 & 1.0 & 1.0 \\
NNW & 0.7 & 1.0 & 1.0 & 1.0 & 1.0 & 0.9 & 1.0 & 1.0 & 1.0 & 1.0 & 1.0 & 1.0 & 1.1 & 0.9 \\
N & 0.6 & 1.0 & 0.9 & 1.0 & 1.0 & 1.0 & 1.0 & 1.0 & 1.0 & 1.0 & 1.0 & 1.0 & 1.1 & 0.7 \\
NNE & 0.6 & 0.9 & 1.0 & 1.0 & 1.0 & 1.0 & 1.0 & 1.0 & 1.0 & 1.0 & 0.8 & 1.0 & 1.1 & 0.9 \\
NE & 0.5 & 1.0 & 1.1 & 1.0 & 1.0 & 0.9 & 0.9 & 1.0 & 1.0 & 1.0 & 0.7 & 1.0 & 1.0 & 0.9 \\
ENE & 1.0 & 1.0 & 1.1 & 1.0 & 1.0 & 1.0 & 1.0 & 1.0 & 1.0 & 1.0 & 1.0 & 1.0 & 1.0 & 1.1 \\
E & 0.9 & 1.0 & 1.0 & 1.0 & 0.9 & 1.0 & 1.0 & 1.0 & 1.0 & 1.0 & 1.0 & 1.0 & 1.0 & 1.0 \\
ESE & 1.0 & 1.0 & 1.0 & 1.0 & 1.0 & 1.0 & 1.0 & 1.0 & 1.0 & 1.0 & 1.0 & 1.0 & 1.0 & 0.9 \\
SE & 1.0 & 1.0 & 1.0 & 1.0 & 1.0 & 1.0 & 1.0 & 1.0 & 1.0 & 1.0 & 1.0 & 1.0 & 1.0 & 1.0 \\
SSE & 1.0 & 1.0 & 1.0 & 1.0 & 0.9 & 1.0 & 1.0 & 1.0 & 1.0 & 1.0 & 1.0 & 1.0 & 1.0 & 0.9 \\
\hline
\end{tabular}


Table 5. Relative Air Concentrations At Offsite Locations Corresponding To Table 2 (Highest Highlighted)

\begin{tabular}{|c|c|c|c|c|c|c|c|c|c|c|c|c|c|c|c|}
\hline & & APT & & er & & Area & F-Area & H-Area & a & & M-Area & N- & P-Area & S/Z-Area & T-Area \\
\hline $\mathbf{S}$ & (E-09 & $8 \mathrm{E}-09$ & $2.23 \mathrm{E}-09$ & $32 \mathrm{E}-09$ & $7.97 \mathrm{E}-09$ & $74 \mathrm{E}-09$ & $1.31 \mathrm{E}-09$ & $1.84 \mathrm{E}-09$ & $2.81 \mathrm{E}-09$ & $3.63 \mathrm{E}-09$ & $.61 \mathrm{E}-09$ & $1.83 \mathrm{E}-09$ & 53E-09 & $1.75 \mathrm{E}-09$ & \\
\hline W & 08 & .09 & -09 & -09 & 2.23E-08 & E-09 & 6.77E-09 & $5.13 \mathrm{E}-09$ & $67 \mathrm{E}-09$ & $5.97 \mathrm{E}-09$ & $1.49 \mathrm{E}-08$ & $6.27 \mathrm{E}-09$ & $36 \mathrm{E}-09$ & 09 & \\
\hline$w$ & .08 & -09 & 08 & 08 & $4.65 \mathrm{E}-08$ & -08 & $2.01 \mathrm{E}-08$ & $1.00 \mathrm{E}-08$ & 03E-08 & $1.58 \mathrm{E}-08$ & 74E-08 & $1.57 \mathrm{E}-08$ & 1.42E-08 & -09 & \\
\hline$V$ & $84 \mathrm{E}-08$ & -09 & 98 & 09 & .08 & $E-09$ & $2.25 \mathrm{E}-08$ & 39E-09 & $.62 \mathrm{E}-08$ & $1.23 \mathrm{E}-08$ & $7.28 \mathrm{E}-08$ & $1.17 \mathrm{E}-08$ & 08 & -09 & 08 \\
\hline W & .08 & 09 & 1.30 & -09 & 2.5 & -09 & $E-08$ & 22E-09 & .08 & 8.04E-09 & E-08 & $E-08$ & 6.3 & 09 & -08 \\
\hline JW & -07 & -08 & 08 & -09 & -08 & 8-09 & -08 & -09 & 09 & $6.29 \mathrm{E}-09$ & -08 & -09 & 4.9 & 09 & -08 \\
\hline & -07 & 08 & $1.08 \mathrm{E}-08$ & E-09 & 2.81E-08 & E-08 & $1.46 \mathrm{E}-08$ & .09 & -08 & 7.30 & 8.7 & 09 & .09 & 08 & -08 \\
\hline NW & -07 & $8 \mathrm{E}-08$ & 1.44E-08 & $13 \mathrm{E}-09$ & $3.22 \mathrm{E}-08$ & 33E-08 & $1.84 \mathrm{E}-08$ & -08 & .08 & 09 & 1. & 08 & .09 & 08 & 08 \\
\hline $\mathbf{N}$ & -07 & -08 & $1.26 \mathrm{E}-08$ & $1 \mathrm{E}-08$ & 9.76E-09 & 49E-08 & $1.75 \mathrm{E}-08$ & $1.36 \mathrm{E}-08$ & 09 & 8. & 7 & 08 & 09 & & 08 \\
\hline JE & 7.50E-08 & -08 & 9.48E-09 & E-09 & $6.96 \mathrm{E}-09$ & E-08 & $1.18 \mathrm{E}-08$ & -08 & -09 & 9.3 & 8 & 99 & 1. & 88 & 09 \\
\hline $\mathbf{N}$ & $6.69 \mathrm{E}-08$ & E-08 & 9.80E-09 & $8.79 \mathrm{E}-09$ & 4.99E-09 & $9.90 \mathrm{E}-09$ & $1.24 \mathrm{E}-08$ & -08 & 09 & 9.9 & 08 & 08 & 08 & 88 & 09 \\
\hline NE & $1.49 \mathrm{E}-08$ & 4E-08 & 9.40E-09 & $1.02 \mathrm{E}-08$ & $6.17 \mathrm{E}-09$ & (9.28E-09 & 1.08E-08 & $1.05 \mathrm{E}-08$ & $1.04 \mathrm{E}-08$ & 1.5 & 08 & 08 & 08 & 8 & \\
\hline $\mathbf{E}$ & 09 & 08 & 1.05E-08 & $8.99 \mathrm{E}-09$ & 7.47E-09 & $6.14 \mathrm{E}-09$ & 9.66E-09 & -09 & E-09 & 1.28 & -09 & 08 & 08 & 09 & \\
\hline ESE & 4.8 & -09 & $7.02 \mathrm{E}-09$ & $6.25 \mathrm{E}-09$ & 9.81E-09 & 5.97E-09 & 7.64E-09 & $6.91 \mathrm{E}-09$ & $1.06 \mathrm{E}-08$ & 1.22 & E-09 & 09 & 08 & 09 & 09 \\
\hline SE & 2.5 & -09 & 4.77E-09 & -09 & 9.06E-09 & 3.4 & 3.87E-09 & 3.70E-09 & 8.85E-09 & 8.37 & -09 & 4.96 & 08 & 09 & 9 \\
\hline SSE & & & 3.80 & -09 & $1.07 \mathrm{E}-08$ & 2.98E-09 & 2.73 & $3.09 \mathrm{E}-09$ & $6.53 \mathrm{E}-09$ & $6.11 \mathrm{E}-09$ & $2.05 \mathrm{E}-09$ & $3.91 \mathrm{E}-09$ & $6.45 \mathrm{E}-09$ & -09 & \\
\hline
\end{tabular}


Table 6. Relative Air Concentrations At Offsite Locations Corresponding To Table 3 - (Highest Highlighted)

\begin{tabular}{|c|c|c|c|c|c|c|c|c|c|c|c|c|c|c|c|}
\hline & A-Area & APT & C-Area & Center & D-Area & E-Area & F-Area & H-Area & K-Area & L-Area & M-Area & N-Area & P-Area & S/Z-Area & T-Area \\
\hline$S$ & $1.54 \mathrm{E}-09$ & $1.78 \mathrm{E}-09$ & $2.24 \mathrm{E}-09$ & 2.32E-09 & $7.97 \mathrm{E}-09$ & $1.74 \mathrm{E}-09$ & $1.31 \mathrm{E}-09$ & $1.84 \mathrm{E}-09$ & $2.81 \mathrm{E}-09$ & $3.63 \mathrm{E}-09$ & $1.61 \mathrm{E}-09$ & $1.83 \mathrm{E}-09$ & $3.53 \mathrm{E}-09$ & $1.75 \mathrm{E}-09$ & $1.14 \mathrm{E}-08$ \\
\hline SSW & 1.33E-08 & $5.72 \mathrm{E}-09$ & 7.14E-09 & $6.14 \mathrm{E}-09$ & $2.23 \mathrm{E}-08$ & 5.79E-09 & $6.77 \mathrm{E}-09$ & 5.13E-09 & 9.67E-09 & 5.97E-09 & 1.49E-08 & $6.27 \mathrm{E}-09$ & $8.86 \mathrm{E}-09$ & $5.01 \mathrm{E}-09$ & 2.66E-08 \\
\hline SW & $5.78 \mathrm{E}-08$ & $9.70 \mathrm{E}-09$ & $1.78 E-08$ & $1.15 E-08$ & $4.65 \mathrm{E}-08$ & $1.11 \mathrm{E}-08$ & $2.01 \mathrm{E}-08$ & $1.00 \mathrm{E}-08$ & $2.03 \mathrm{E}-08$ & $1.58 \mathrm{E}-08$ & 5.74E-08 & $1.57 \mathrm{E}-08$ & $1.42 \mathrm{E}-08$ & $9.82 \mathrm{E}-09$ & $5.35 \mathrm{E}-08$ \\
\hline WSW & 8.84E-08 & $9.69 \mathrm{E}-09$ & 1.33E-08 & 7.32E-09 & 3.73E-08 & $9.85 \mathrm{E}-09$ & $2.25 \mathrm{E}-08$ & 8.39E-09 & $1.62 \mathrm{E}-08$ & $1.23 \mathrm{E}-08$ & 7.28E-08 & $1.17 \mathrm{E}-08$ & $1.03 \mathrm{E}-08$ & $9.36 \mathrm{E}-09$ & $4.96 \mathrm{E}-08$ \\
\hline W & $8.59 \mathrm{E}-08$ & $8.43 \mathrm{E}-09$ & $1.30 \mathrm{E}-08$ & 7.49E-09 & $2.59 \mathrm{E}-08$ & 9.94E-09 & $1.40 \mathrm{E}-08$ & 8.22E-09 & $1.05 \mathrm{E}-08$ & $8.04 \mathrm{E}-09$ & 7.46E-08 & $1.13 \mathrm{E}-08$ & $6.32 \mathrm{E}-09$ & 8.40E-09 & $3.62 \mathrm{E}-08$ \\
\hline WNW & $1.10 \mathrm{E}-07$ & 1.12E-08 & $1.12 \mathrm{E}-08$ & 7.44E-09 & $1.98 \mathrm{E}-08$ & $9.10 \mathrm{E}-09$ & $1.15 \mathrm{E}-08$ & $8.41 \mathrm{E}-09$ & $9.89 \mathrm{E}-09$ & $6.29 \mathrm{E}-09$ & $6.26 \mathrm{E}-08$ & 9.59E-09 & $4.92 \mathrm{E}-09$ & $9.38 \mathrm{E}-09$ & $2.89 \mathrm{E}-08$ \\
\hline NW & $1.38 \mathrm{E}-07$ & $1.58 \mathrm{E}-08$ & $1.08 \mathrm{E}-08$ & 7.74E-09 & $2.81 \mathrm{E}-08$ & $1.13 \mathrm{E}-08$ & $1.46 \mathrm{E}-08$ & $9.83 E-09$ & $1.29 \mathrm{E}-08$ & 7.30E-09 & $8.70 \mathrm{E}-08$ & $9.44 E-09$ & $5.73 E-09$ & $1.07 \mathrm{E}-08$ & $3.86 \mathrm{E}-08$ \\
\hline NNW & $1.55 \mathrm{E}-07$ & $1.98 \mathrm{E}-08$ & $1.44 \mathrm{E}-08$ & 9.13E-09 & $3.22 \mathrm{E}-08$ & $1.33 \mathrm{E}-08$ & $1.84 \mathrm{E}-08$ & $1.25 \mathrm{E}-08$ & $1.10 \mathrm{E}-08$ & $8.07 \mathrm{E}-09$ & 1.32E-07 & $1.32 \mathrm{E}-08$ & 7.35E-09 & $1.22 \mathrm{E}-08$ & $4.41 \mathrm{E}-08$ \\
\hline $\mathrm{N}$ & $1.51 \mathrm{E}-07$ & 3.01E-08 & $1.26 \mathrm{E}-08$ & 1.10E-08 & $9.76 \mathrm{E}-09$ & $1.49 \mathrm{E}-08$ & $1.75 \mathrm{E}-08$ & $1.36 \mathrm{E}-08$ & $9.53 \mathrm{E}-09$ & 9.06E-09 & $1.06 \mathrm{E}-07$ & $1.19 \mathrm{E}-08$ & -08 & 1.58 & -08 \\
\hline NNE & 7.50E-08 & 2.23E-08 & $1.04 \mathrm{E}-08$ & $9.18 \mathrm{E}-09$ & $6.16 \mathrm{E}-09$ & $1.27 \mathrm{E}-08$ & 1.33E-08 & $1.37 \mathrm{E}-08$ & $8.74 \mathrm{E}-09$ & 9.30E-09 & 5.81E-08 & 1.09E-08 & 1.20E-08 & 1.27E-08 & 8.32E-09 \\
\hline NE & 6.69E-08 & 1.84E-08 & $9.80 \mathrm{E}-09$ & 8.79E-09 & $4.99 \mathrm{E}-09$ & $9.90 \mathrm{E}-09$ & 1.24E-08 & $1.06 \mathrm{E}-08$ & 7.01E-09 & $9.96 \mathrm{E}-09$ & $4.67 \mathrm{E}-08$ & $1.02 \mathrm{E}-08$ & 1.42E-08 & $1.10 \mathrm{E}-08$ & E-09 \\
\hline ENE & $1.69 \mathrm{E}-08$ & -08 & $9.40 \mathrm{E}-09$ & $1.02 \mathrm{E}-08$ & $6.17 \mathrm{E}-09$ & -09 & $1.08 \mathrm{E}-08$ & $1.05 \mathrm{E}-08$ & $1.04 \mathrm{E}-08$ & $1.54 \mathrm{E}-08$ & $1.62 \mathrm{E}-08$ & $1.04 \mathrm{E}-08$ & -08 & $1.06 \mathrm{E}-08$ & $4.82 \mathrm{E}-09$ \\
\hline E & 8.04E-09 & $1.03 \mathrm{E}-08$ & $1.05 \mathrm{E}-08$ & 8.99E-09 & 7.47E-09 & $6.14 \mathrm{E}-09$ & $9.66 \mathrm{E}-09$ & 7.05E-09 & $9.67 \mathrm{E}-09$ & $1.28 \mathrm{E}-08$ & 7.41E-09 & $1.19 \mathrm{E}-08$ & $1.8 \mathrm{~s}$ & $6.99 \mathrm{E}-09$ & -09 \\
\hline ESE & 4.83E-09 & 8.98E-09 & 7.02E-09 & $6.25 \mathrm{E}-09$ & $9.81 \mathrm{E}-09$ & 5.97E-09 & 7.64E-09 & $6.91 \mathrm{E}-09$ & $1.06 \mathrm{E}-08$ & 1.22E-08 & 4.88E-09 & 7.66E-09 & -08 & $6.71 \mathrm{E}-09$ & -09 \\
\hline SE & $2.58 \mathrm{E}-09$ & $5.15 \mathrm{E}-09$ & $4.77 \mathrm{E}-09$ & $4.51 \mathrm{E}-09$ & $9.06 \mathrm{E}-09$ & $3.40 \mathrm{E}-09$ & $3.87 \mathrm{E}-09$ & $3.70 \mathrm{E}-09$ & $8.85 \mathrm{E}-09$ & 8.37 & -09 & $4.96 \mathrm{E}-09$ & 1.0 & 3.57E-09 & 7.98E-09 \\
\hline SSE & 2.00E-09 & $3.88 \mathrm{E}-09$ & $3.80 \mathrm{E}-09$ & 3.83E-09 & $1.07 \mathrm{E}-08$ & $2.98 \mathrm{E}-09$ & $2.73 \mathrm{E}-09$ & $3.09 \mathrm{E}-09$ & $6.53 \mathrm{E}-09$ & $6.11 \mathrm{E}-09$ & 2.05E-09 & -09 & 6.45E-09 & 2.98E-09 & 1.08E-08 \\
\hline
\end{tabular}


SREL Conference Center, respectively. Since relative air concentration is directly proportional to dose, the concentrations for each sector and distance are compared to determine which is the highest. The maximum concentration has been highlighted. Table 7 shows a summary of the worst sector and distance for each of the potential release locations both considering and not considering the SREL Conference Center. The numbers in parentheses refer to input required for CAP88.

Table 7. Location of MEI for NESHAPS Calculations

\begin{tabular}{|c|c|c|c|c|c|r|}
\hline & \multicolumn{2}{|c|}{ Offsite MEI } & \multicolumn{2}{c|}{ SREL Included } & \multicolumn{2}{c|}{ Offsite MEI } \\
\hline Area & Sector & Distance & Sector & Distance & Sector & Distance \\
\hline A-Area & NNW(2) & 970 & NNW(2) & 970 & NNW(2) & 1360 \\
APT & $\mathrm{N}(1)$ & 9480 & $\mathrm{~N}(1)$ & 8370 & $\mathrm{~N}(1)$ & 9350 \\
C-Area & $\mathrm{SW}(7)$ & 12750 & $\mathrm{SW}(7)$ & 12750 & $\mathrm{SW}(7)$ & 13100 \\
Center & $\mathrm{SW}(7)$ & 16200 & $\mathrm{SW}(7)$ & 16200 & $\mathrm{SW}(7)$ & 16420 \\
D-Area & $\mathrm{SW}(7)$ & 5000 & $\mathrm{SW}(7)$ & 5000 & $\mathrm{WSW}(6)$ & 6640 \\
E-Area & $\mathrm{N}(1)$ & 11480 & $\mathrm{~N}(1)$ & 11480 & $\mathrm{~N}(1)$ & 11050 \\
F-Area & $\mathrm{WSW}(6)$ & 10230 & $\mathrm{WSW}(6)$ & 10230 & $\mathrm{SW}(7)$ & 15230 \\
H-Area & $\mathrm{N}(1)$ & 12380 & $\mathrm{NNE}(16)$ & 11460 & $\mathrm{~N}(1)$ & 12370 \\
K-Area & $\mathrm{SW}(7)$ & 10990 & $\mathrm{SW}(7)$ & 10990 & $\mathrm{SW}(7)$ & 11920 \\
L-Area & $\mathrm{SW}(7)$ & 12510 & $\mathrm{SW}(7)$ & 12510 & $\mathrm{ENE}(14)$ & 12670 \\
M-Area & $\mathrm{NNW(2)}$ & 1340 & $\mathrm{NNW(2)}$ & 1340 & $\mathrm{NNW}(2)$ & 1370 \\
N-Area & $\mathrm{SW}(7)$ & 14130 & $\mathrm{SW}(7)$ & 14130 & $\mathrm{SW}(7)$ & 14320 \\
P-Area & $\mathrm{ENE}(14)$ & 8470 & $\mathrm{ENE}(14)$ & 8470 & $\mathrm{ENE}(14)$ & 8450 \\
S/Z-Area & $\mathrm{N}(1)$ & 11800 & $\mathrm{~N}(1)$ & 10940 & $\mathrm{~N}(1)$ & 10970 \\
T-Area & $\mathrm{SW}(7)$ & 4450 & $\mathrm{SW}(7)$ & 4450 & $\mathrm{WSW}(6)$ & 4270 \\
\hline
\end{tabular}

\section{ANALYSIS OF RESULTS}

Table 8 shows the ratio of distances determined in this study to distances determined in 1990 . Looking at Table 8, noticeable differences are seen for the following locations: A Area, D Area, and $F$ Area. If the ratio shown in the last column of Table 8 is less than one, the resulting dose could increase since the MEI is now closer to the release location. Calculations were performed for each of the areas to demonstrate the magnitude of the differences.

Table 9 shows the comparison of relative air concentrations using 1990 distances versus 1999 distances. Relative air concentration is directly proportional to dose so this table represents potential dose differences that would be seen using the new distances. The largest difference is seen in D Area. This is due to the fact that not only did the distance between the MEI and the release location decrease, but the worst sector changed. 
Table 8. Comparison of Distances Determined in 1999 and 1990

\begin{tabular}{|c|c|c|c|c|c|}
\hline & \multicolumn{2}{|c|}{ Offsite MEI } & \multicolumn{2}{c|}{$\begin{array}{c}\text { Offsite MEI } \\
1990\end{array}$} & $\begin{array}{c}\text { Ratio Dist } \\
1999 / 1990\end{array}$ \\
\hline Area & Sector & Distance & Sector & Distance & \\
\hline A-Area & NNW(2) & 970 & NNW(2) & 1360 & 0.7 \\
APT & N(1) & 9480 & N(1) & 9350 & 1.0 \\
C-Area & SW(7) & 12750 & SW(7) & 13100 & 1.0 \\
Center & SW(7) & 16200 & SW(7) & 16420 & 1.0 \\
D-Area & SW(7) & 5000 & WSW(6) & 6640 & 0.8 \\
E-Area & N(1) & 11480 & N(1) & 11050 & 1.0 \\
F-Area & WSW(6) & 10230 & SW(7) & 15230 & 0.7 \\
H-Area & N(1) & 12380 & N(1) & 12370 & 1.0 \\
K-Area & SW(7) & 10990 & SW(7) & 11920 & 0.9 \\
L-Area & SW(7) & 12510 & ENE(14) & 12670 & 1.0 \\
M-Area & NNW(2) & 1340 & NNW(2) & 1370 & 1.0 \\
N-Area & SW(7) & 14130 & SW(7) & 14320 & 1.0 \\
P-Area & ENE(14) & 8470 & ENE(14) & 8450 & 1.0 \\
S/Z-Area & N(1) & 11800 & N(1) & 10970 & 1.1 \\
T-Area & SW(7) & 4450 & WSW(6) & 4270 & 1.0 \\
\hline
\end{tabular}

Table 9. Comparison of Relative Air Concentrations using 1999 and 1990 Distances

\begin{tabular}{|l|c|c|c|}
\hline & $\begin{array}{c}\mathbf{1 9 9 9} \\
\text { Distance }\end{array}$ & $\begin{array}{c}\mathbf{1 9 9 0} \\
\text { Distance }\end{array}$ & $\begin{array}{c}\% \\
\text { Difference }\end{array}$ \\
\hline AREA & Chi/Q & Chi/Q & \\
\hline A & $1.55 \mathrm{E}-07$ & $1.31 \mathrm{E}-07$ & $18 \%$ \\
APT & $2.52 \mathrm{E}-08$ & $2.57 \mathrm{E}-08$ & $-2 \%$ \\
$\mathbf{C}$ & $1.78 \mathrm{E}-08$ & $1.72 \mathrm{E}-08$ & $3 \%$ \\
CENTER & $1.15 \mathrm{E}-08$ & $1.13 \mathrm{E}-08$ & $2 \%$ \\
$\mathbf{D}$ & $4.65 \mathrm{E}-08$ & $3.16 \mathrm{E}-08$ & $47 \%$ \\
$\mathbf{E}$ & $1.49 \mathrm{E}-08$ & $1.56 \mathrm{E}-08$ & $-4 \%$ \\
$\mathbf{F}$ & $2.25 \mathrm{E}-08$ & $2.03 \mathrm{E}-08$ & $11 \%$ \\
$\mathbf{H}$ & $1.36 \mathrm{E}-08$ & $1.36 \mathrm{E}-08$ & $0 \%$ \\
$\mathbf{K}$ & $2.03 \mathrm{E}-08$ & $1.84 \mathrm{E}-08$ & $10 \%$ \\
$\mathbf{L}$ & $1.58 \mathrm{E}-08$ & $1.56 \mathrm{E}-08$ & $1 \%$ \\
$\mathbf{M}$ & $1.32 \mathrm{E}-07$ & $1.30 \mathrm{E}-07$ & $2 \%$ \\
$\mathbf{P}$ & $2.33 \mathrm{E}-08$ & $2.33 \mathrm{E}-08$ & $0 \%$ \\
S & $1.44 \mathrm{E}-08$ & $1.57 \mathrm{E}-08$ & $-8 \%$ \\
$\mathbf{T}$ & $5.35 \mathrm{E}-08$ & $5.36 \mathrm{E}-08$ & $0 \%$ \\
\hline
\end{tabular}




\section{CONCLUSIONS}

Distances to the maximally exposed offsite individual have been determined for demonstrating NESHAPS compliance. Should the SREL Conference Center become a potential offsite location, distances also have been included for this facility. Three release locations (APT, H, S/Z) would show increases in dose predictions if the SREL Conference Center were considered. 


\section{REFERENCES}

Beres, Deborah A. 'The Clean Air Act Assessment Package - 1988 (CAP88) A Dose and Risk Assessment Methodology For Radionuclide Emissions To Air, Volume 1, User's Manual,' U.S. Environmental Protection Agency, Washington, D.C. 1990.

EPA 1989 U.S. Environmental Protection Agency, 1989, "National Emission Standards for Hazardous Air Pollutants; Radionuclides," Title 40 Code of Federal Regulations, Part 61 , Volume 54, No 240, Washington, D.C.

Mackey, Hal. “NESHAPS Distances Summary Memo, Tables, and Figures (U)” Westinghouse Inter-Office Memorandum: PECD-EGIS-99-0035, September 13, 1999, Westinghouse Savannah River Company, Aiken, SC. 
WESTINGHOUSE SAVANNAH RIVER COMPANY

APPENDIX A. Mackey's NESHAPS Distance Memo 


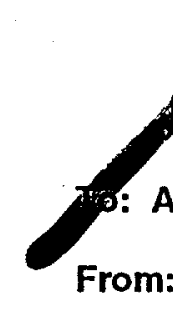

Ali Simpkins, SRTC, 773-42A, RM228

Sim

NESHAPS Distances Summary Memo, Tables, and Figures (U)
PECD-EGIS-99-0035

September 13, 1999

Attached are Tables and Figures which summarize our efforts to date for estimating the distances from 15 points (source locations-- A, APT, C, Center of SRS, D, E, F, H, K, L, M, N, P, $S Z, T$ ) on the SRS to the nearest offsite feature of interest, i.e., buildings, fields, etc., for the 16 directional sectors from each center point, plus an estimate to the SREL Conference Center. Distances are in meters and coordinates for the off-site locations are in UTMs. In the summary table, we also provide information on the 1996 SRS ortho-photographic tile which was used to identify the off-site feature, its direction, and the type of feature. Site-wide SRS photography from the summer of 1998 , taken by Bechtel Nevada, was also reviewed to verify the off-site features, thus some minor changes were made based on off-site changes from 1996 to 1998 . Figures are provided to show the general location of each feature for each center and sector. Note, a number of the off-site features are used several times for a number of the sectors. At this point in time, we have not conducted off-site visits to verify the identity of the off-site features. Thus, feature selection is based entirely on photographic interpretation. In addition, the final two columns in the summary table are a comparison of the distances from 1989 versus the distances for 1999 (units are kilometers), for those source locations in common for the two years. There is general agreement for most points (a few tenths of a kilometer). However, there are several distances of a few kilometers different. No, attempt has been made to determine if in these latter cases if there were a change in landuse, criteria for off-site feature location, or technical difference in determination of off-site features between our determination in 1999 and in the selections in 1989.

The entire effort has been save as a GIS project, thus both the data base (1996 photography, sectors, etc.) can be review independently, or we would be happy to review the effort and every point selection with you if needed.

This completes the effort at this time. I would recommend that field visits and verification be conducted for at least a sub-set of the off-site points to test the accuracy of the photo interpretation. In addition, a support document to provide QAVQC verification would be in order, so that future updates would be easy and defensible for any NESHAPS based calculation for existing and/or future dose calculations.

CC: EGIS File

John Gladden, SRTC

Russ Beckmeyer, E\&GIS

Larry Koffman, E\&GIS

Cheryl Hardy, E\&GIS 


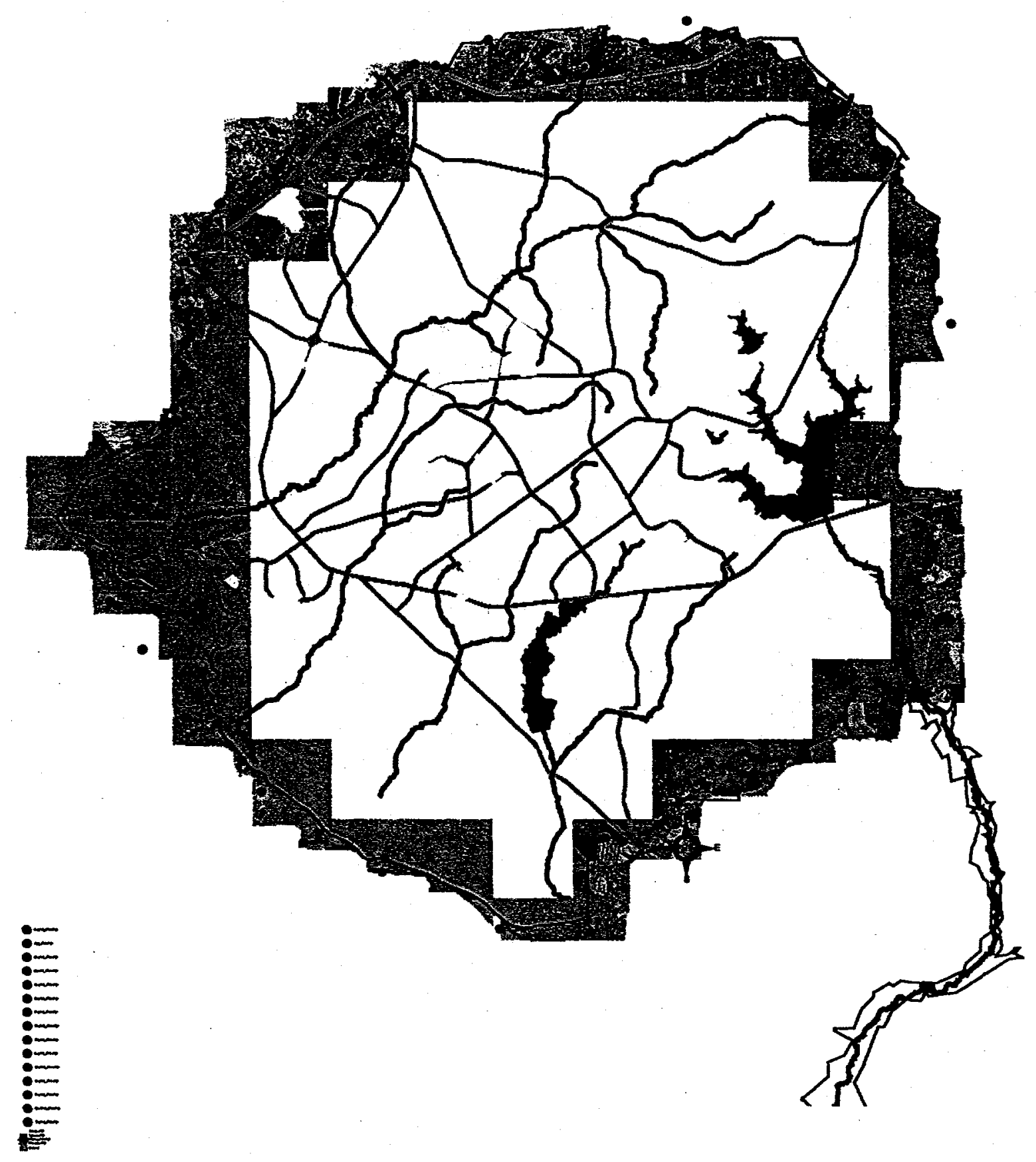

Nearest Offsite Points of Interest

Hal Mackey, Cheryl Hardy, and Larry Koffman -draft-example-9/13/99 


\begin{tabular}{|c|c|c|c|c|c|c|}
\hline AREA & UTM_E & SECTOR & DISTANCE A & AZIMUTH TYPE-COMMENTS & 89Distance & 99distance \\
\hline A-Area & 431264.0 & $3690881.8 \mathrm{~N}$ & 1158.8 & 354.21 Field, D-2 & 1.8 & 1.159 \\
\hline A-Area & 431890.9 & $3692303.1 \mathrm{NNE}$ & 2626.3 & 11.00 Field, D-2 & 1.93 & 2.26 \\
\hline A-Area & 433533.7 & 3692818.3 NE & 3770.0 & 34.60 Field, E-2 & 3.59 & 3.77 \\
\hline A-Area & 443826.5 & 3695499.1 ENE & 13724.8 & 64.79 Field, 9-6468 & 7.93 & 13.725 \\
\hline A-Area & 452104.2 & $3693695.3 \mathrm{E}$ & 21106.5 & 78.82 Building, K-1 & 18.56 & 21.106 \\
\hline A-Area & 453892.7 & 3679167.0 ESE & 24872.4 & 114.78 Building, K-6 & 24.73 & 24.872 \\
\hline A-Area & 448365.2 & $3666787.3 \mathrm{SE}$ & 28548.0 & 143.12 Field, $1-10$ & 26.64 & 28.548 \\
\hline A-Area & 443219.0 & 3664962.5 SSE & 27452.6 & 154.09 Field, $\mathrm{H}-11$ & 22.14 & 27.453 . \\
\hline A-Area & 427401.7 & $3669540.4 \mathrm{~S}$ & 20575.0 & 190.78 Building, C-9 & 13.48 & 20.575 \\
\hline A-Area & 427082.9 & $3683265.0 \mathrm{SSW}$ & 7758.0 & 213.23 Field, C-5 & 5.64 & 7.758 \\
\hline A-Area & 427458.0 & $3687021.2 \mathrm{SW}$ & 4760.6 & 234.99 Building, C-4 & 3.89 & 4.761 \\
\hline A-Area & 428786.2 & 3689210.0 WSW & 2639.1 & 258.33 Field, $\mathrm{C}_{-3}$ & 2.59 & 2.639 \\
\hline A-Area & 429235.6 & $3689828.3 \mathrm{~W}$ & 2140.6 & 272.33 Building, C-3 & 2.15 & 2.141 \\
\hline A-Area & 430265.7 & 3690111.6 WNW & 1172.7 & 288.73 Field, D-2,BN1998 & 1.49 & 1.173 \\
\hline A-Area & 430904.3 & $3690245.4 \mathrm{NW}$ & 698.6 & 317.41 Field, D-2 & 1.47 & 0.699 \\
\hline A-Area & 430912.4 & $3690584.5 \mathrm{NNW}$ & 972.8 & 331.32 Building, D-2 & 1.3 & 0.973 \\
\hline A-Area & 442748.0 & 3694450.7 ENE & 12315.5 & 67.10 Conference, $\mathrm{H}-1$ & & \\
\hline APT & 443837.7 & $3695491.9 \mathrm{~N}$ & 9478.1 & 6.22 Field, photo number & 15.14 & 9.478 \\
\hline APT & 445001.3 & $3695388.8 \mathrm{NNE}$ & 9580.3 & 13.22 Field, $H-1$ & 16.13 & 9.58 \\
\hline APT & 449712.7 & $3695182.6 \mathrm{NE}$ & 11461.2 & 37.04 Field, J-1 & 16.56 & 11.461 \\
\hline APT & 454548.0 & 3690337.1 ENE & 12540.4 & 69.79 Building, L-2 & 15.14 & 12.54 \\
\hline APT & 456150.2 & $3685518.7 \mathrm{E}$ & 13407.9 & 92.03 Building, L-4 & 14.72 & 13.408 \\
\hline APT & 453893.4 & $3679170: 2$ ESE & 13106.4 & 121.45 Building, K-6 & 14.72 & 12.106 \\
\hline APT & 455886.3 & 3676875.6 SE & 16034.6 & 124.66 Field, L-7 & 14.35 & 16.035 \\
\hline APT & 452524.0 & 3670131.6 SSE & 18699.6 & 148.15 Field, K-9 & 14.33 & 18.7 \\
\hline APT & 446387.1 & $3665646.4 \mathrm{~S}$ & 20750.0 & 169.57 Field, $\mid-11$ & 14.33 & 20.75 \\
\hline APT & 430270.5 & 3666713.1 SSW & 23038.0 & 212.46 Building, D-10 & 15.86 & 23.038 \\
\hline APT & 424792.0 & $3673822.9 \mathrm{SW}$ & 21743.1 & 235.35 Field, B-8 & 12.81 & 21.743 \\
\hline APT & 427531.4 & 3682787.0 WSW & 15573.6 & 257.46 Field, C-5 & 12.81 & 15.573 \\
\hline APT & 428351.8 & $3688616.6 \mathrm{~W}$ & 14624.5 & 279.65 Field, C-3 & 12.96 & 14.624 \\
\hline APT & 431479.5 & 3691228.9 WNW & 12396.2 & 294.21 Field, D-2 & 13.2 & 12.396 \\
\hline APT & 437088.9 & $3694550.2 \mathrm{NW}$ & 10193.4 & 325.89 Building, $F-1$ & 14.45 & 10.193 \\
\hline APT & 437408.7 & 3694446.3 NNW & 9931.5 & 327.09 Building, $\mathrm{F}-1$ & 14.45 & 9.931 \\
\hline APT & 442747.9 & $3694450.3 \mathrm{~N}$ & 8374.4 & 359.61 Conference, $\mathrm{H}-1$ & & \\
\hline C-Area & 434174.3 & $3693299.5 \mathrm{~N}$ & 14474.6 & 348.44 Field, Photo Number & & \\
\hline C-Area & 443825.8 & 3695493.4 NNE & 17761.4 & 22.29 Field, 1-1 & & \\
\hline C-Area & 448711.8 & $3695363.2 \mathrm{NE}$ & 20047.6 & 35.44 Building, J-1 & & \\
\hline C-Area & 456151.3 & 3685518.7 ENE & 20209.4 & 71:13 Building, L-4 & & \\
\hline C-Area & 453892.2 & $3679168.6 \mathrm{E}$ & 16904.4 & 89.42 Building, K-6 & & \\
\hline C-Area & 452523.4 & 3670132.1 ESE & 17938.5 & 119.65 Field, K-9 & & \\
\hline C-Area & 446387.1 & 3665644.7 SE & 16413.8 & 144.72 Field, $1-11$ & & \\
\hline C-Area & 443219.5 & $3664963.6 \mathrm{SSE}$ & 15449.8 & 155.86 Field, $\mathrm{H}-11$ & & \\
\hline C-Area & 434920.2 & $3664141.2 \mathrm{~S}$ & 15102.0 & 187.52 Building, E-11 & & \\
\hline C-Area & 429505.9 & 3667892.4 SSW & 13476.5 & 213.37 Building, D-10 & & \\
\hline C-Area & 427484.4 & $3670599.8 \mathrm{SW}$ & 12751.0 & 227.84 Building, C-9 & & \\
\hline C-Area & 425727.6 & 3672145.0 WSW & 13235.7 & 237.94 Field, B-8 & & \\
\hline C-Area & 426668.1 & $3680933.5 \mathrm{~W}$ & 10481.4 & 279.72 Field, B-6 & & \\
\hline C-Area & 427531.0 & 3682786.1 WNW & 10149.6 & 290.94 Fiold, C-5 & & \\
\hline C-Area & 427092.0 & $3685717.5 \mathrm{NW}$ & 11904.2 & 303.42 Field, C-4 & & \\
\hline C-Area & 430903.6 & 3690240.3 NNW & 12692.7 & 331.01 Field, D-2? & & \\
\hline C-Area & 442747.0 & $3694451.1 \mathrm{NNE}$ & 16394.9 & 20.22 Conference, $\mathrm{H}-1$ & & \\
\hline Center & 439050.1 & $3695529.9 \mathrm{~N}$ & 15813.3 & 352.94 Building, $\mathrm{H}-1$ & 16.1 & 15.813 \\
\hline Center & 444021.6 & 3695503.8 NNE & 15987.4 & 10.92 Field, $\mathrm{H}-1$ & 16.26 & 15.987 \\
\hline Center & 454548.1 & $3690334.9 \mathrm{NE}$ & 17228.2 & 52.06 Building, $L-2$ & 17.55 & 17.228 \\
\hline Center & 456152.0 & 3685519.0 ENE & 16282.9 & 69.18 Building, L-4 & 16.58 & 16.283 \\
\hline Center & 453892.4 & $3679167.4 \mathrm{E}$ & 13011.8 & 92.55 Building, K-6 & 13.2 & 13.012 \\
\hline Center & 455392.4 & 3675142.9 ESE & 15232.6 & 107.55 Field, L-7 & 15.3 & 15.233 \\
\hline Center & 452518.8 & $3670135.0 \mathrm{SE}$ & 15131.2 & 129.47 Field, K-9 & 15.3 & 15.131 \\
\hline Center & 448362.0 & 3666785.9 SSE & 15024.5 & 149.86 Field, J-10 & 15.3 & 15.024 \\
\hline Center & 443328.6 & $3664990.1 \mathrm{~S}$ & 15032.5 & 170.34 Field, $\mathrm{H}-11$ & 15.13 & 15.032 \\
\hline Center & 434920.5 & 3664141.1 SSW & 16783.9 & 200.51 Building, E-11 & 17.23 & 16.784 \\
\hline Center & 428567.9 & $3669311.4 \mathrm{SW}$ & 16203.2 & 229.20 Building, C-9 & 16.42 & 16.203 \\
\hline Center & 425728.1 & 3672142.5 WSW & 17003.5 & 242.79 Field, B-8 & 17.23 & 17.004 \\
\hline
\end{tabular}




\begin{tabular}{|c|c|c|c|c|c|c|}
\hline Center & 427323.7 & $3682521.1 \mathrm{~W}$ & 13838.7 & 280.89 Field, C-5 & 14.17 & 13.839 \\
\hline Center & 427531.4 & 3682787.1 WNW & 13690.1 & 282.15 Field, C-5 & 13.85 & 13.69 \\
\hline Center & 430904.2 & $3690240.2 \mathrm{NW}$ & 14434.0 & 315.84 Field, D-2 & 14.65 & 14.434 \\
\hline Center & 437408.4 & 3694446.0 NNW & 15031.7 & 346.23 Building, photo number & 15.3 & 15.032 \\
\hline Center & 442747.0 & $3694450.2 \mathrm{~N}$ & 14741.9 & 6.86 Conference, $\mathrm{H}-1$ & & \\
\hline D-Area & 428321.2 & $3688577.4 \mathrm{~N}$ & 14867.9 & 348.57 Building, C-3 & 9.52 & 14.868 \\
\hline D-Area & 436279.4 & 3694588.7 NNE & 21223.9 & 13.55 Field, photo number & 16.92 & 21.224 \\
\hline D-Area & 446623.9 & $3696213.4 \mathrm{NE}$ & 27065.9 & 34.44 Building, photo number & 23.87 & 27.066 \\
\hline D-Area & 453892.8 & 3679167.0 ENE & 23296.9 & 76.80 Building, K-6 & 24.3 & 23.297 \\
\hline D-Area & 452522.9 & $3670132.6 \mathrm{E}$ & 21688.9 & 99.89 Field, K-9 & 18.63 & 21.689 \\
\hline D-Area & 445173.9 & 3664688.5 ESE & 16802.0 & 123.25 Field, $1-11$ & 14.87 & 16.802 \\
\hline D-Area & 443192.4 & $3664951.1 \mathrm{SE}$ & 15032.0 & 126.60 Field, $\mathrm{H}-11$ & 10.38 & 15.032 \\
\hline D-Area & 434920.9 & 3664140.4 SSE & 10533.3 & 158.84 Building, E-11 & 6.97 & 10.533 \\
\hline D-Area & 430269.9 & $3666714.2 \mathrm{~S}$ & 7329.1 & 186.78 Building, D-10 & 5.34 & 7.329 \\
\hline D-Area & 428567.9 & $3669311.5 \mathrm{SSW}$ & 5355.0 & 208.84 Building, C-9 & 3.39 & 5.355 \\
\hline D-Area & 427480.7 & $3670627.8 \mathrm{sW}$ & 4996.1 & 227.41 Building, C-9 & 2.79 & 4.996 \\
\hline D-Area & 425692.2 & 3672131.6 WSW & 5792.0 & 250.97 Field, B-8? & 1.66 & 5.792 \\
\hline D-Area & 424791.7 & $3673822.5 \mathrm{~W}$ & 6389.6 & 268.18 Field, B-8 & 1.66 & 6.389 \\
\hline D-Area & 423726.5 & 3676322.2 WNW & 7810.0 & 287.05 Field, A-7 & 2.64 & 7.81 \\
\hline D-Area & 425582.7 & $3679953.2 \mathrm{NW}$ & 8180.4 & 316.48 Field, B-6 & 3.76 & 8.18 \\
\hline D-Area & 426652.3 & 3680933.1 NNW & 8291.3 & 326.56 Field, B-6 & 8.01 & 8.291 \\
\hline D-Area & 442747.2 & 3694450.2 NNE & 23506.9 & 29.13 Conference, $\mathrm{H}-1$ & & \\
\hline E-Area & 437408.8 & $3694446.9 \mathrm{~N}$ & 11478.1 & 353.86 Building, Photo Number & & \\
\hline E-Area & 443831.3 & 3695495.3 NNE & 13534.2 & 22.55 Field, $H-1$ & & \\
\hline E-Area & 447651.7 & 3695705.4 NE & 15597.6 & 35.28 Field, $1-1$ & & \\
\hline E-Area & 454549.3 & 3690334.8 ENE & 17574.6 & 65.08 Building, L-2 & & \\
\hline E-Area & 456151.3 & $3685518.0 \mathrm{E}$ & 17761.0 & 81.59 Building, $L-4$ & & 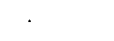 \\
\hline E-Area & 453892.7 & 3679165.8 ESE & 15805.7 & 103.79 Building, $\mathrm{K}-6$ & & \\
\hline E-Area & 452522.3 & $3670132.0 \mathrm{SE}$ & 19001.8 & 132.39 Fiold, K-9 & & \\
\hline E-Area & 443210.4 & 3664964.8 SSE & 18650.1 & 165.23 Field, $\mathrm{H}-11$ & & \\
\hline E-Area & 434920.5 & $3664140.5 \mathrm{~S}$ & 19235.3 & 190.57 Building, E-11 & & \\
\hline E-Area & 429504.4 & 3667895.7 SSW & 17637.3 & 210.57 Building, $\mathrm{C}-10$ & & \\
\hline E-Area & 424797.9 & $3673817.9 \mathrm{SW}$ & 16564.1 & 235.87 Field, B-8 & & \\
\hline E-Area & 425605.4 & 3679970.2 WSW & 13316.1 & 256.38 Field, B-6 & & \\
\hline E-Area & 427678.7 & $3684345.6 \mathrm{~W}$ & 10966.7 & 276.55 Field, C-4. & & \\
\hline E-Area & 428834.7 & 3689261.2 WNW & 11556.7 & 302.29 Field, C-3 & & \\
\hline E-Area & 430906.0 & $3690245.9 \mathrm{NW}$ & 10525.3 & 312.95 Field, D-2 & & \\
\hline E-Area & 433535.4 & 3692817.8 NNW & 11007.3 & 332.46 Field, E-2 & & \\
\hline E-Area & 442747.0 & 3694450.8 NNE & 12164.7 & 19.75 Conference, $\mathrm{H}-1$ & & \\
\hline F-Area & 434974.0 & $3693786.6 \mathrm{~N}$ & 10933.3 & 348.80 Field, Photo Number & 10.29 & 10.933 \\
\hline F-Area & 443830.3 & 3695492.1 NNE & 14179.7 & 28.30 Field, $\mathrm{H}-1$ & 11.35 & 14.18 \\
\hline F-Area & 446619.5 & $3696218.1 \mathrm{NE}$ & 16290.0 & 35.71 Building, Photo Number & 14 & 16.29 \\
\hline F-Area & 454549.1 & $3690336.1 \mathrm{ENE}$ & 18973.0 & 67.06 Building, $L-2$ & 17.49 & 18.973 \\
\hline F-Area & 456151.9 & $3685519.0 \mathrm{E}$ & 19279.4 & 82.29 Building, $L-4$ & 18.8 & 19.279 \\
\hline F-Area & 453892.8 & 3679166.4 ESE & 17302.5 & 102.62 Building, K-6 & 18.8 & 17.302 \\
\hline F-Area & 448364.8 & 3666788.1 SE & 19820.4 & 144.77 Field, $\mathrm{J}-10$ & 18.98 & 19.82 \\
\hline F-Area & 443212.1 & 3664960.6 SSE & 19114.8 & 160.78 Field, $\mathrm{H}-11$ & 18.96 & 19.115 \\
\hline F-Area & 434921.0 & $3664140.2 \mathrm{~S}$ & 19025.9 & 186.02 Building, E-11 & 16.93 & 19.026 \\
\hline F-Area & 428567.3 & $3669312.0 \mathrm{SSW}$ & 16135.0 & 211.29 Building, C-9 & 11.85 & 16.135 \\
\hline F-Area & 424793.0 & $3673820.2 \mathrm{SW}$ & 15327.9 & 232.63 Field, B-8 & 11.49 & 15.328 \\
\hline F-Area & 427016.3 & 3680970.7 WSW & 10228.5 & 257.93 Field, $\mathrm{C}-6$ & 9.39 & 10.228 \\
\hline F-Area & 427678.8 & $3684344.0 \mathrm{~W}$ & 9441.9 & 277.54 Field, $\mathrm{C}_{-4}$ & 9.39 & 9.442 \\
\hline F-Area & 427227.9 & 3685002.6 WNW & 9996.4 & 280.92 Field, C-4 & 9.4 & 9.996 \\
\hline F-Area & 430904.7 & $3690242.9 \mathrm{NW}$ & 9449.6 & 319.23 Field, D-2 & 9.43 & 9.45 \\
\hline F-Area & 431510.1 & 3691323.4 NNW & 9947.8 & 325.94 Field, E-2 & 9.43 & 9.948 \\
\hline F-Area & 442746.8 & 3694450.4 NNE & 12754.0 & 26.27 Conference, $\mathrm{H}-1$ & & \\
\hline H-Area & 439049.6 & $3695529.0 \mathrm{~N}$ & 12379.7 & 353.17 Building, Photo Number & 11.54 & 12.38 \\
\hline H-Area & 443830.6 & 3695492.9 NNE & 12722.6 & 15.07 Field, $\mathrm{H}-1$ & 12.59 & 12.723 \\
\hline H-Area & 448711.5 & $3695363.3 \mathrm{NE}$ & 14681.8 & 33.91 Building, J-1 & 13.08 & 14.682 \\
\hline H-Area & 454549.0 & $3690335.9 \mathrm{ENE}$ & 15791.7 & 62.90 Building, L-2 & 15.35 & 15.792 \\
\hline H-Area & 456152.7 & $3685517.2 \mathrm{E}$ & 15871.5 & 81.36 Building, L-4 & 15.47 & 15.872 \\
\hline H-Area & 453893.2 & 3679166.6 ESE & 14046.2 & 106.46 Building, K-6 & 15.52 & 14.046 \\
\hline H-Area & 452524.0 & $3670131.9 \mathrm{SE}$ & 17814.8 & 136.97 Field, K-9 & 16.44 & 17.815 \\
\hline H-Area & 450086.1 & 3667934.0 SSE & 18078.4 & 147.43 Field, J-10 & 17.71 & 18.078 \\
\hline
\end{tabular}




\begin{tabular}{|c|c|c|c|c|c|c|}
\hline H-Area & 443321.0 & $3664987.8 \mathrm{~S}$ & 18465.6 & 170.70 Field, $\mathrm{H}-11$ & 17.85 & 18.466 \\
\hline H-Area & 430270.6 & 3666714.0 SSW & 19398.2 & 211.29 Building, D-10 & 16.67 & 19.398 \\
\hline H-Area & 427480.3 & $3670628.3 \mathrm{SW}$ & 18080.4 & 225.47 Building, C-9 & 14.34 & 18.08 \\
\hline H-Area & 425606.7 & 3679968.9 WSW & 15194.2 & 257.26 Field, B-6 & 12.81 & 15.194 \\
\hline H-Area & 427678.8 & $3684344.0 \mathrm{~W}$ & 12817.0 & 274.64 Field, C-4 & 12.79 & 12.817 \\
\hline H-Area & 430108.5 & 3689975.5 WNW & 12345.5 & 302.78 Field, D-3 & 11.83 & 12.345 \\
\hline H-Area & 433535.4 & $3692817.3 \mathrm{NW}$ & 11820.2 & 323.87 Field, E-2 & 11.74 & $\$ 1.82$ \\
\hline H-Area & 437408.9 & 3694447.3 NNW & 11623.0 & 344.50 Building, $F-1$ & 11.54 & 11.623 \\
\hline H-Area & 442747.2 & $3694450.5 \mathrm{NNE}$ & 11455.4 & 11.23 Conference, $\mathrm{H}-1$ & & \\
\hline K-Area & 434671.0 & $3693514.0 \mathrm{~N}$ & 19026.9 & 349.40 Field, Photo Number & 17.9 & 19.027 \\
\hline K-Area & 443825.5 & 3695493.2 NNE & 21491.3 & 15.22 Field, $H-1$ & 20.57 & 21.491 \\
\hline K-Area & 454548.6 & $3690335.3 \mathrm{NE}$ & 22663.2 & 46.34 Building, L-2 & 19.36 & 22.663 \\
\hline K-Area & 453893.4 & 3679167.7 ENE & 16430.6 & 74.20 Building, K-6 & 17.1 & 16.431 \\
\hline K-Area & 455393.9 & $3675145.2 \mathrm{E}$ & 17341.0 & 88.48 Field, L-7 & 14.78 & 17.341 \\
\hline K-Area & 450086.1 & 3667933.0 ESE & 13847.0 & 119.34 Field, J-10 & 11.64 & 13.847 \\
\hline K-Area & 445969.7 & $3665469.5 \mathrm{SE}$ & 12227.1 & 139.32 Field, $1-11$ & 10.76 & 12.227 \\
\hline K-Area & 443202.7 & 3664958.5 SSE & 11097.6 & 152.02 Field, $H-11 ?$ & 10.76 & 11.098 \\
\hline K-Area & 439284.3 & $3661446.1 \mathrm{~S}$ & 13401.0 & 174.39 Field, G-12 & 10.95 & 13.401 \\
\hline K-Area & 434920.3 & 3664140.7 SSW & 11102.1 & 196.06 Building, E-11 & 10.58 & 11.102 \\
\hline K-Area & 429505.2 & $3667893.7 \mathrm{SW}$ & 10986.1 & 230.76 Building, C-10 & 10.58 & 10.986 \\
\hline K-Area & 428567.8 & 3669311.8 WSW & 10957.0 & 239.65 Building, C-9 & 8.86 & 10.957 \\
\hline K-Area & 424795.8 & $3673819.6 \mathrm{~W}$ & 13296.2 & 265.46 Field, B-8 & 8.86 & 13.296 \\
\hline K-Area & 426607.9 & 3680760.6 WNW & 12911.7 & 297.19 Field, Photo Number & 9.64 & 12.912 \\
\hline K-Area & 427458.6 & $3682680.3 \mathrm{NW}$ & 13212.2 & 306.32 Field, C-5 & 12.42 & 13.212 \\
\hline K-Area & 430905.0 & 3690240.5 NNW & 17024.7 & 334.81 Field, D-2 & 15.81 & 17.025 \\
\hline K-Area & 442747.2 & 3694450.3 NNE & 20211.5 & 13.07 Conference, $\mathrm{H}-1$ & & \\
\hline L-Area & 443841.3 & $3695490.3 \mathrm{~N}$ & 21068.8 & 4.87 Field, $\mathrm{H}-1$ & 20.34 & 21.069 \\
\hline L-Area & 453653.4 & 3692375.4 NNE & 21372.1 & 32.93 Building, $\mathrm{K}-2$ & 19.92 & 21.372 \\
\hline L-Area & 456641.2 & $3684642.5 \mathrm{NE}$ & 17867.6 & 55.10 Building, L-4 & 15.27 & 17.868 \\
\hline L-Area & 453893.6 & 3679167.5 ENE & 12843.2 & 68.38 Building, K-6 & 13.42 & 12.843 \\
\hline L-Area & 455392.7 & $3675145.9 \mathrm{E}$ & 13482.5 & 86.94 Field, Photo Number & 11.42 & 13.482 \\
\hline L-Area & 452020.7 & 3669242.9 ESE & 11386.4 & 117.20 Building, K-9 & 10.25 & 11.386 \\
\hline L-Area & 448364.4 & 3666787.8 SE & 10053.8 & 139.82 Field, J-10 & 9.16 & 10.054 \\
\hline L-Area & 443992.4 & 3664820.6 SSE & 9906.3 & 167.61 Building, $\mathrm{H}-11$ & 9.16 & 9.906 \\
\hline L-Area & 443323.1 & $3664990.4 \mathrm{~S}$ & 9620.5 & 171.29 Field, $\mathrm{H}-11$. & 9.5 & 9.62 \\
\hline L-Area & 434844.4 & 3663641.9 SSW & 12970.3 & 212.74 Field, E-11 & 12.09 & 12.97 \\
\hline L-Area & 434921.4 & $3664140.0 \mathrm{SW}$ & 12512.6 & 213.69 Building, E-11 & 12.09 & 12.513 \\
\hline L-Area & 429502.4 & 3667895.0 WSW & 14073.7 & 241.62 Building, C-10 & 12.5 & 14.074 \\
\hline L-Area & 425721.2 & $3672137.3 \mathrm{~W}$ & 16376.6 & 261.32 Field, B-8 & 12.5 & 16.377 \\
\hline L-Area & 426984.7 & 3681382.2 WNW & 16445.7 & 294.35 Field, C-5 & 13.24 & 16.446 \\
\hline L-Area & 427648.8 & $3684101.1 \mathrm{NW}$ & 17199.6 & 303.55 Field, C-4 & 15.77 & 17.2 \\
\hline L-Area & 431264.9 & 3690883.4 NNW & 19538.9 & 326.58 Field, D-2 & 19.18 & 19.539 \\
\hline L-Area & 442747.0 & $3694450.6 \mathrm{~N}$ & 19958.7 & 2.01 Conference, $\mathrm{H}-1$ & & \\
\hline M-Area & 431261.0 & $3690881.0 \mathrm{~N}$ & 1932.4 & -0.19 Field, D-2 & 1.8 & 1.932 \\
\hline M-Area & 431924.3 & 3692272.0 NNE & 3390.1 & 11.03 Field, E-2 & 1.93 & 3.39 \\
\hline M-Area & 434146.5 & 3693270.9 NE & 5200.0 & 33.43 Field, Photo Number & 3.59 & 5.2 \\
\hline M-Area & 443830.4 & 3695492.6 ENE & 14175.7 & 62.16 Field, $\mathrm{H}-1$ & 7.98 & 14.176 \\
\hline M-Area & 453653.6 & $3692373.9 \mathrm{E}$ & 22658.5 & 80.96 Building, $\mathrm{K}-2$ & 18.56 & 22.658 \\
\hline M-Area & 453894.4 & 3679167.6 ESE & 24661.4 & 113.02 Building, $\mathrm{K}-6$ & 24.73 & 24.661 \\
\hline M-Area & 448364.1 & 3666786.8 SE & 27997.3 & 141.98 Field, $\mathrm{J}-10$ & 26.64 & 27.997 \\
\hline M-Area & 443209.5 & 3664961.9 SSE & 26800,4 & 153.16 Field, $H-11$ & 22.14 & 26.8 \\
\hline M-Area & 427401.6 & $3669539.1 \mathrm{~S}$ & 19788.4 & 190.88 Building, C-9 & 13.48 & 19.788 \\
\hline M-Area & 427329.4 & 3683060.7 SSW & 7076.9 & 213.35 Field, C-5 & 5.64 & 7.077 \\
\hline M-Area & 427256.6 & $3686266.3 \mathrm{SW}$ & 4815.2 & 235.80 Field, C-4 & 3.89 & 4.815 \\
\hline M-Area & 428180.8 & 3688329.3 WSW & 3136.5 & 258.26 Field, C-3 & 2.59 & 3.136 \\
\hline M-Area & 428836.7 & $3689267.4 \mathrm{~W}$ & 2439.8 & 277.16 Field, $\mathrm{C}-3$ & 2.15 & 2.44 \\
\hline M-Area & 429236.1 & 3689828.3 WNW & 2202.8 & 293.19 Building, C-3 & 1.49 & 2.203 \\
\hline M-Area & 430098.8 & $3689961.5 \mathrm{NW}$ & 1537.6 & 310.86 Field, $D-3$ & 1.47 & 1.538 \\
\hline M-Area & 430904.8 & $3690244.1 \mathrm{NNW}$ & 1342.2 & 344.50 Field, D-2 & 1.3 & 1.342 \\
\hline M-Area & 442747.1 & 3694450.4 ENE & 12740.7 & 64.07 Conference, $\mathrm{H}-1$ & & \\
\hline N-Area & 437408.4 & $3694446.6 \mathrm{~N}$ & 15187.7 & 355.02 Building. Photo Number & & \\
\hline N-Area & 443824.4 & 3695493.2 NNE & 16996.4 & 17.43 Field, $H-1$ & & \\
\hline N-Area & 449869.0 & $3695043.5 \mathrm{NE}$ & 19333.9 & 35. 18 Field, $J-1$ & & \\
\hline $\mathrm{N}$-Area & 456151.1 & $3685517.8 \mathrm{ENE}$ & 18584.4 & 70.13 Building, L-4 & & \\
\hline
\end{tabular}




\begin{tabular}{|c|c|c|c|c|c|c|}
\hline N-Area & 453893.6 & $3679166.6 \mathrm{E}$ & 15259.5 & 90.18 Building, K-6 & & \\
\hline N-Area & 452521.4 & 3670133.2 ESE & 16644.0 & 123.10 Field, K-9 & & \\
\hline $\mathrm{N}$-Area & 448362.5 & $3666788.3 \mathrm{SE}$ & 15855.1 & 141.80 Fiold, J-10 & & \\
\hline N-Area & 443230.6 & 3664968.3 SSE & 15058.6 & 161.88 Field, H-11 & & \\
\hline N-Area & 439286.0 & $3661446.4 \mathrm{~S}$ & 17874.0 & 177.56 Field, G-12 & & \\
\hline $\mathrm{N}$-Area & 430270.1 & 3666713.8 SSW & 15119.3 & 213.24 Building, D-10 & & \\
\hline N-Area & 427480.0 & $3670627.9 \mathrm{SW}$ & 14134.4 & 231.76 Building, C-9 & & \\
\hline $\mathrm{N}$-Area & 425708.4 & 3672130.1 WSW & 14785.8 & 240.61 Field, B-8 & & \\
\hline N-Area & 426986.1 & $3681385.0 \mathrm{~W}$ & 11832.2 & 279.76 Field, C-5 & & \\
\hline N-Area & 427532.1 & 3682784.4 WNW & 11634.0 & 287.03 Field, $\mathrm{C}-5$ & & \\
\hline N-Area & 430903.6 & $3690239.5 \mathrm{NW}$ & 13388.7 & 324.38 Field, Photo Number & & \\
\hline N-Area & 431263.9 & $3690884.5 \mathrm{NNW}$ & 13723,4 & 327.16 Field, Photo Number & & \\
\hline N-Area & 442747.0 & 3694450.3 NNE & 15690.3 & 14.84 Conference, $\mathrm{H}-1$ & & \\
\hline P-Area & 448712.0 & $3695363.1 \mathrm{~N}$ & 19021.0 & 8.35 Building, J-1 & 17.88 & 19.021 \\
\hline P-Area & 454682.5 & $3690111.1 \mathrm{NNE}$ & 16182.4 & 32.79 Field, L-2 & 14.52 & 16.182 \\
\hline P-Area & 456640.6 & 3684642.5 NE & 13492.6 & 52.85 Building, L-4 & 11.04 & 13.493 \\
\hline P-Area & 453892.9 & 3679167.1 ENE & 8467.8 & 71.72 Building, K-6 & 9.71 & 8.468 \\
\hline P-Area & 455395.0 & $3675149.8 \mathrm{E}$ & 9662.4 & 98.05 Field, $L-7$ & 9.59 & 9.662 \\
\hline P-Area & 455482.7 & 3674592.1 ESE & 9845.3 & 101.18. Field, L-8 & 9.21 & 9.845 \\
\hline P-Area & 452516.5 & 3670136.1 SE & 9268.3 & 133.53 Field, K-9 & 9.21 & 9.268 \\
\hline P-Area & 450085.3 & 3667933.4 SSE & 9616.9 & 153.43 Field, J-10 & 9.47 & 9.617 \\
\hline P-Area & 446386.7 & $3665646.5 \mathrm{~S}$ & 10928.0 & 176.76 Fiold, $1-11$ & 9.87 & 10.928 \\
\hline P-Area & 443379.9 & 3664990.8 SSW & 11827.8 & 191.64 Field, $H-11$ & 10.42 & 11.828 \\
\hline P-Area & 434921.4 & $3664140.4 \mathrm{SW}$ & 16534.6 & 220.96 Building, E-11 & 15.68 & 16.535 \\
\hline P-Area & 430270.2 & 3666713.6 WSW & 18419.2 & 237.33 Building, D-10 & 16.68 & 18.419 \\
\hline P-Area & 425607.1 & $3679969.5 \mathrm{~W}$ & 20514.3 & 279.22 Field, B-6 & 16.68 & 20.514 \\
\hline P-Area & 426984.8 & 3681384.1 WNW & 19458.6 & 284.00 Field, $\mathrm{C}-6$ & 18.8 & 19.459 \\
\hline P-Area & 430904.5 & $3690241.7 \mathrm{NW}$ & 20251.5 & 312.15 Field, D-2 & 19.67 & 20.251 \\
\hline P-Area & 437408.8 & 3694446.7 NNW & 19772.0 & 334.42 Building. Photo Number & 18.72 & 19.772 \\
\hline P-Area & 4427.47 .6 & $3694450.6 \mathrm{~N}$ & 18154.6 & 349.85 Conference, $\mathrm{H}-1$ & * & \\
\hline SZ-Area & 439049.8 & $3695528.7 \mathrm{~N}$ & 11794.9 & 353.71 Building. Photo Number & & \\
\hline SZ-Area & 443840.2 & 3695489.1 NNE & 12224.7 & 16.63 Field, $\mathrm{H}-1$ & & \\
\hline SZ-Area & 448711.5 & $3695363.5 \mathrm{NE}$ & 14318.9 & 35.77 Building, $J-1$ & & \\
\hline SZ-Area & 454548.5 & 3690334.3 ENE & 15703.2 & 65.05 Building, L-2 & & \\
\hline SZ-Area & 456151.3 & $3685518.2 \mathrm{E}$ & 15973.7 & 83.46 Building, L-4 & & \\
\hline SZ-Area & 453894.5 & 3679166.9 ESE & 14389.2 & 108.42 Building, K-6 & & \\
\hline SZ-Area & 452521.0 & 3670133.8 SE & 18351.0 & 137.77 Field, K-9 & & \\
\hline SZ-Area & 450086.5 & 3667932.6 SSE & 18655.7 & 147.90 Field, $\mathrm{J}-10$ & & \\
\hline SZ-Area & 443324.5 & $3664989.2 \mathrm{~S}$ & 19054.2 & 170.43 Field, $\mathrm{H}-11$ & & \\
\hline SZ-Area & 430270.1 & 3666714.0 SSW & 19795.0 & 209.99 Building, D-10 & & \\
\hline SZ-Area & 427479.9 & $3670627.8 \mathrm{SW}$ & 18358.5 & 223.81 Building, C-9 & & \\
\hline SZ-Area & 426674.6 & 3680939.3 WSW & 13893.2 & 257.78 Fieid, B-6 & & \\
\hline SZ-Area & 427679.1 & $3684345.2 \mathrm{~W}$ & 12603.4 & 272.14 Fieid, C-4 & & \\
\hline SZ-Area & 433534.2 & $3692818.0 \mathrm{NW}$ & 11258.6 & 322.90 Field, E-2 & & \\
\hline SZ-Area & 437409.0 & 3694446.5 NNW & 11027.2 & 344.61 Building, $F-1$ & & \\
\hline SZ-Area & 430273.7 & 3690117.9 WNW & 11827.5 & 301.95 Field, D-2 & & \\
\hline SZ-Area & 442747.0 & $3694450.3 \mathrm{~N}$ & 10937.2 & 12.74 Conference, $\mathrm{H}-1$ & & \\
\hline T-Area & 427449.8 & $3683846.1 \mathrm{~N}$ & 9116.7 & 348.48 Field, $\mathrm{C}-4$ & & \\
\hline T-Area & 433536.2 & 3692818.4 NNE & 18429.7 & 13.21 Field, Photo Number & & \\
\hline T-Area & 443831.1 & $3695496.8 \mathrm{NE}$ & 25253.4 & 35.01 Field, $\mathrm{H}-1$ & & \\
\hline T-Area & 456150.5 & 3685517.7 ENE & 28950.8 & 68.14 Building, $L-4$ & & \\
\hline T-Area & 453893.2 & $3679167.5 \mathrm{E}$ & 25042.9 & 79.84 Building, K-6 & & \\
\hline T-Area & 444648.2 & 3664710.0 ESE & 18493.9 & 123.09 Building, $\mathrm{H}-11$ & & \\
\hline T-Area & 439286.0 & $3661444.5 \mathrm{SE}$ & 16807.9 & 142.84 Field, G-12 & & \\
\hline T-Area & 431843.9 & 3664758.9 SSE & 10478.0 & 165.13 Field, D-11 & & \\
\hline T-Area & 428567.7 & $3669312.1 \mathrm{~S}$ & 5627.5 & 186.27 Bullding, C-9 & & \\
\hline T-Area & 427480.2 & 3670627.8 SSW & 4613.4 & 201.75 Bullding, C-9 & & \\
\hline T-Area & 425726.6 & 3672140.5 SW & 4450.1 & 231.29 Field, B-8 & & \\
\hline T-Area & 424795.2 & 3673821.0 WSW & 4551.3 & 255.91 Field, B-8 & & \\
\hline T-Area & 424489.5 & $3673981.0 \mathrm{~W}$ & 4815.7 & 258.62 Field, B-8 & & \\
\hline T-Area & 423727.5 & 3676322.9 WNW & 5669.7 & 284.16 Field, A-7 & & \\
\hline T-Area & 425592.6 & $3679958.5 \mathrm{NW}$ & 6220.7 & 324.02 Field, B-6 & & \\
\hline T-Area & 426595.1 & 3680733.9 NNW & 6393.7 & 335.45 Fieid, B-6 & & \\
\hline T-Area & 442747.0 & $3694450.2 \mathrm{NE}$ & 23774.2 & 34.34 Conference, $\mathrm{H}-1$ & & \\
\hline
\end{tabular}




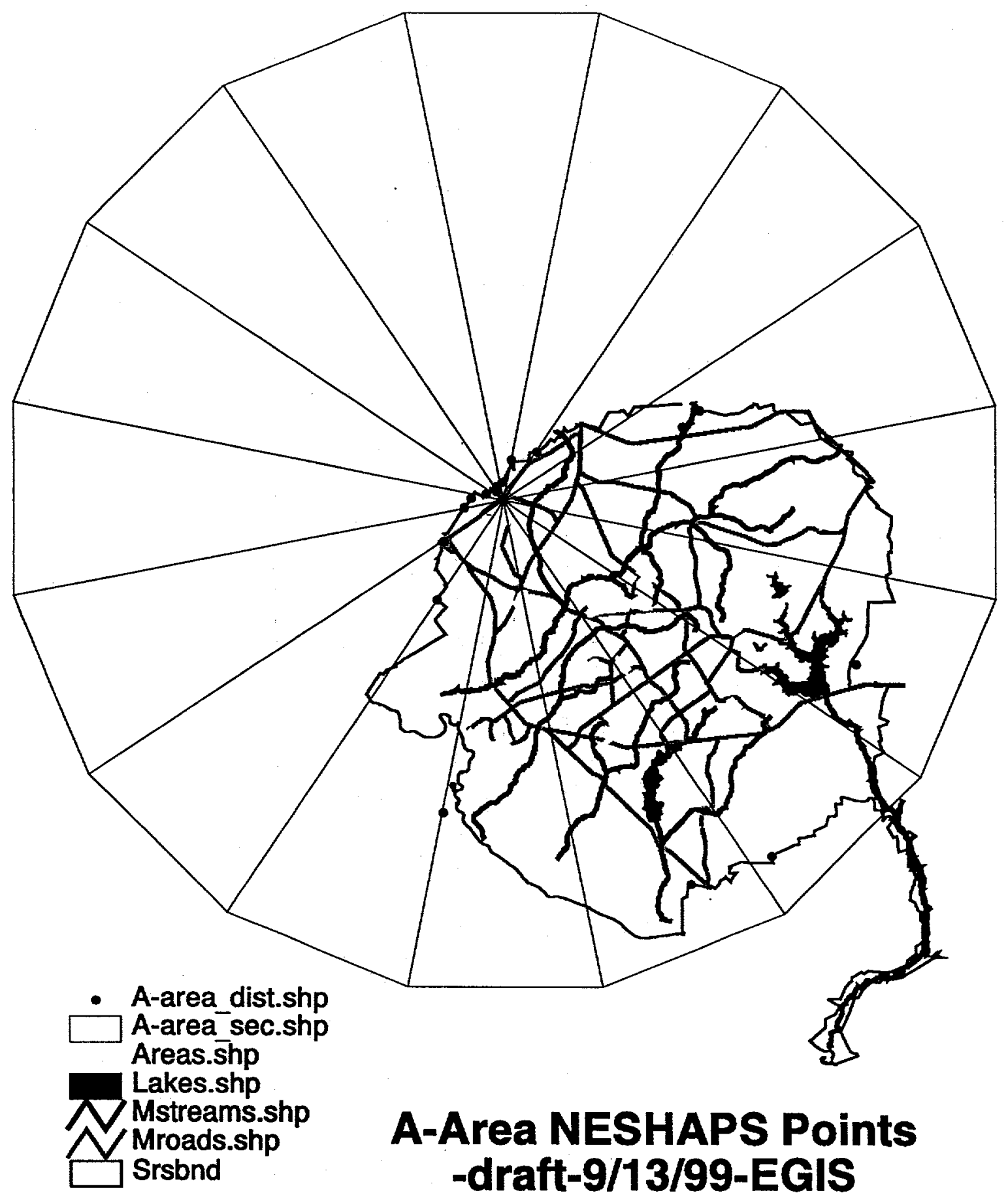




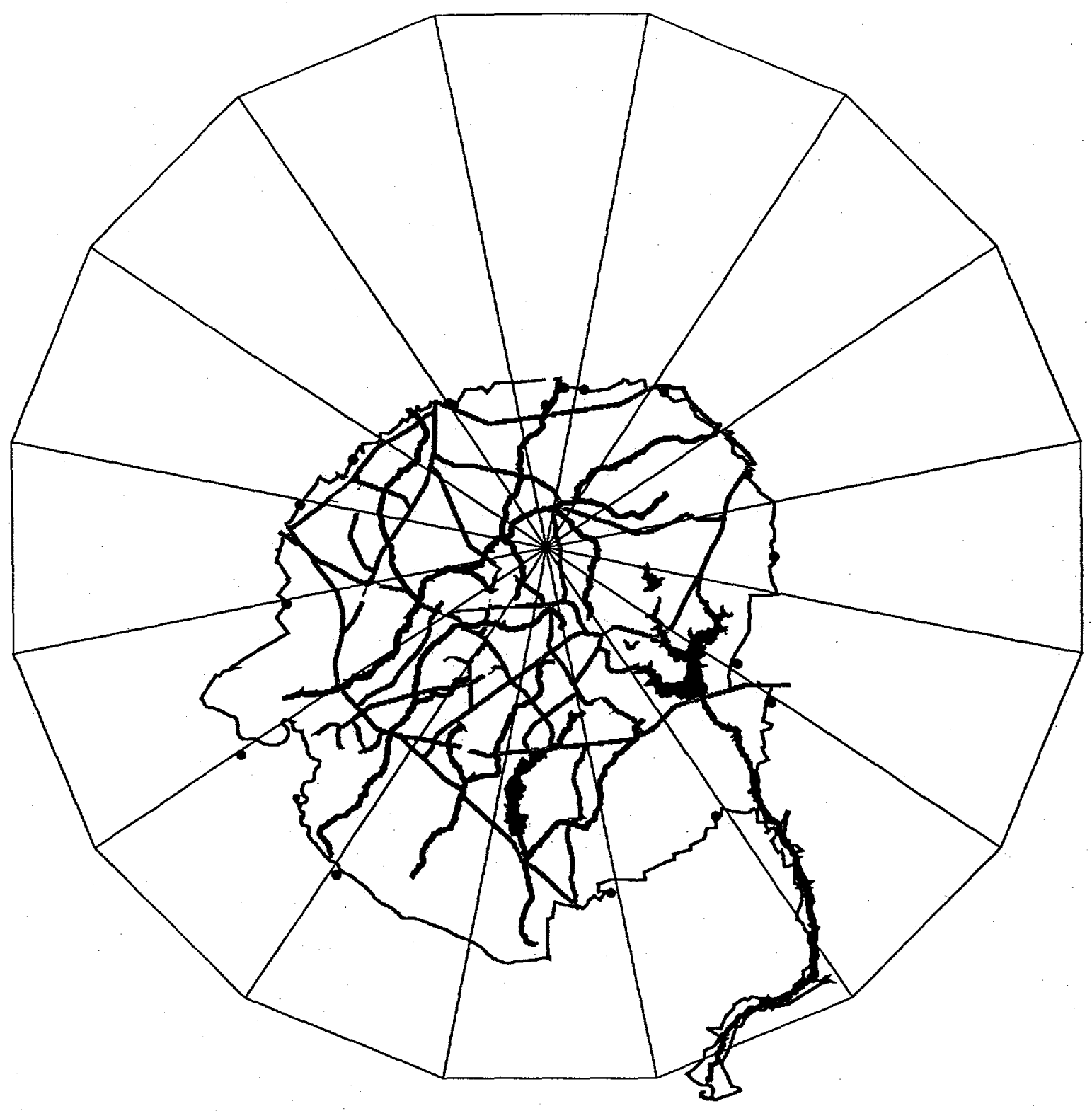

- Apt dist.shp

$\square$ Apt_sec.shp Areas.shp

Lakes.shp

Mstreams.shp

APT NESHAPS Points

-draft-9/13/99-EGIS

Mroads.shp

Srsbnd 


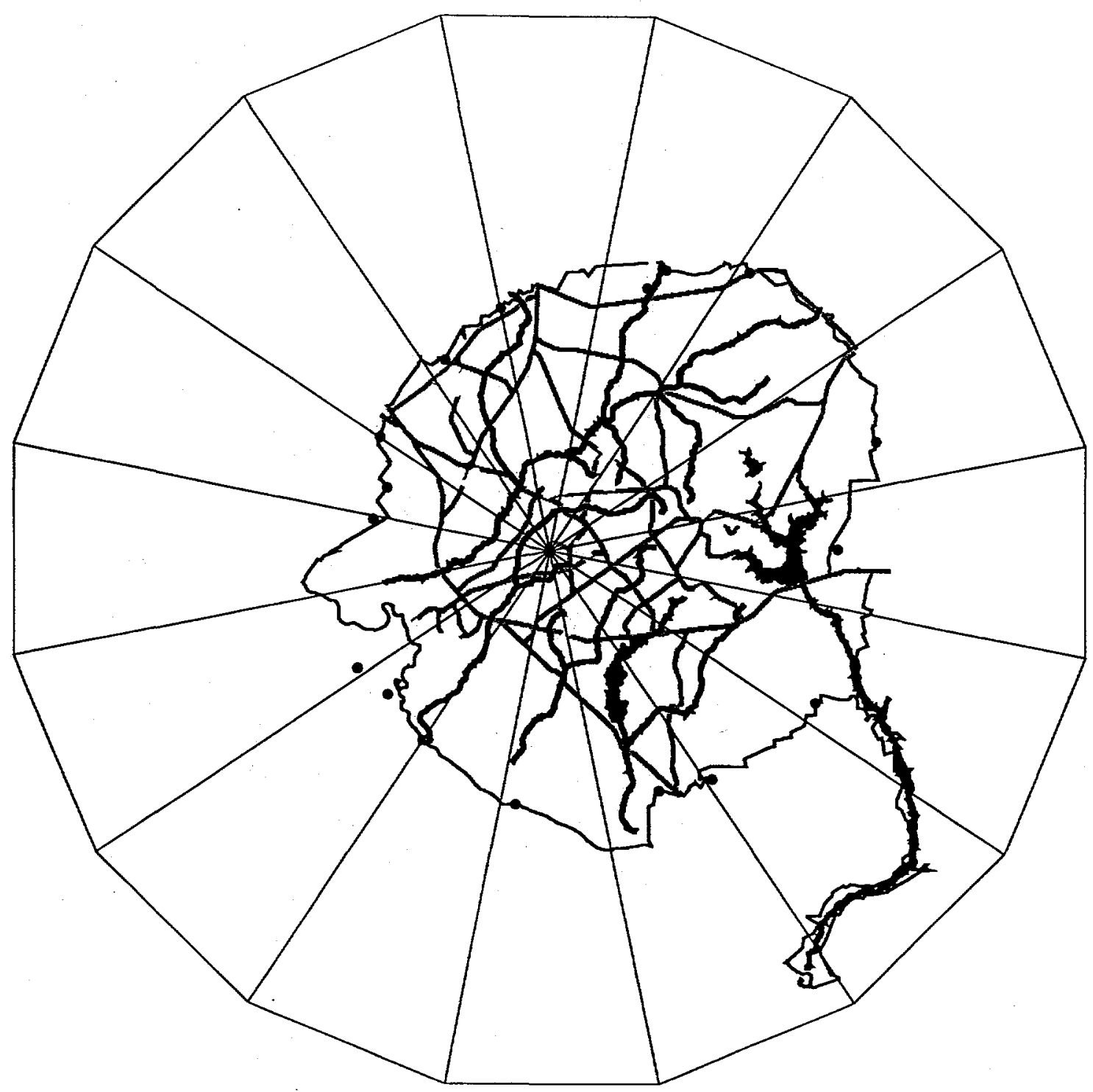

- C-area_dist.shp

$\square$ C-area sec.shp

Areas.shp

Lakes.shp
Mstreams.shp
Mroads.shp
Srsbnd

C-Area NESHAPS Points -draft-9/13/99-EGIS 


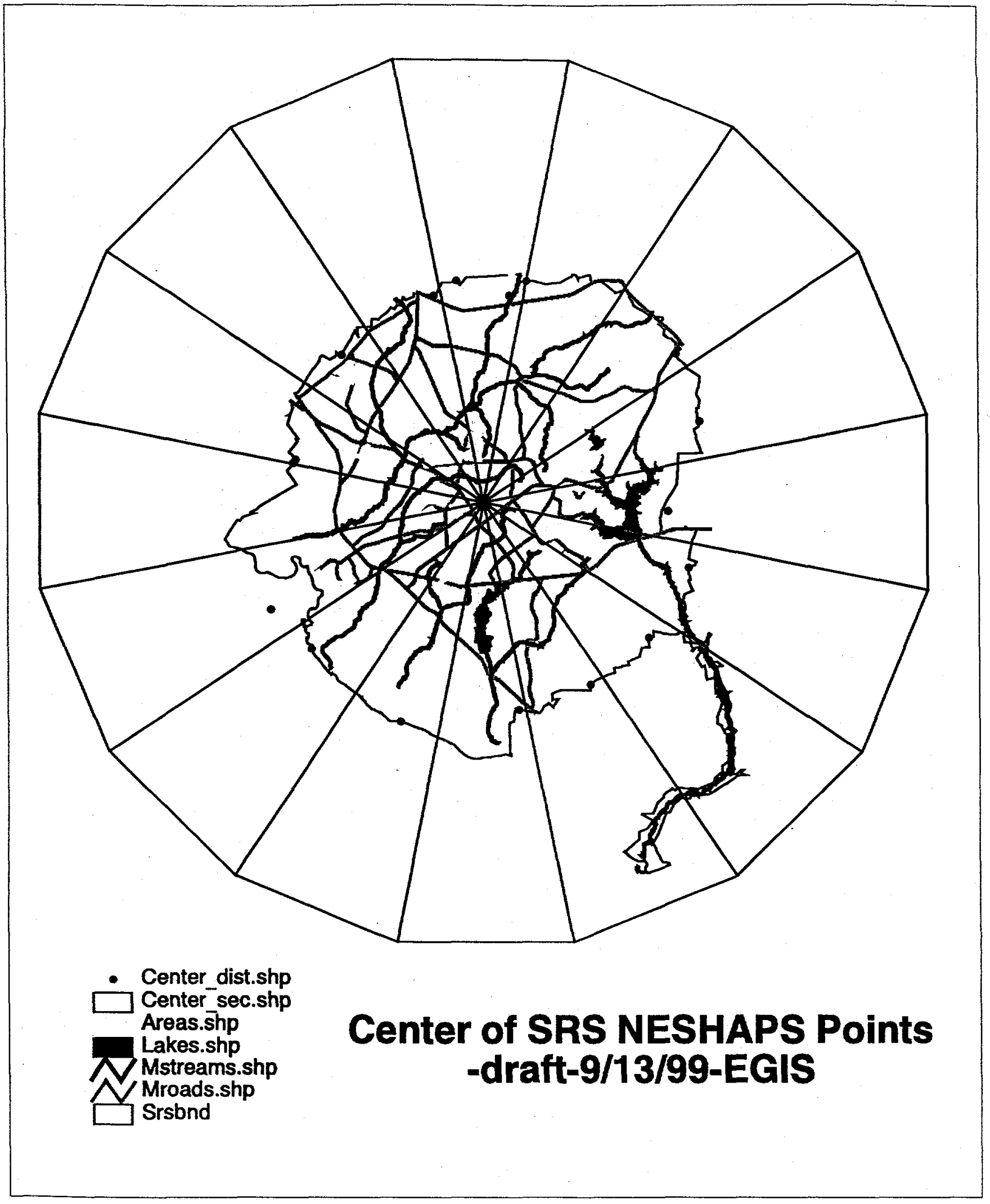




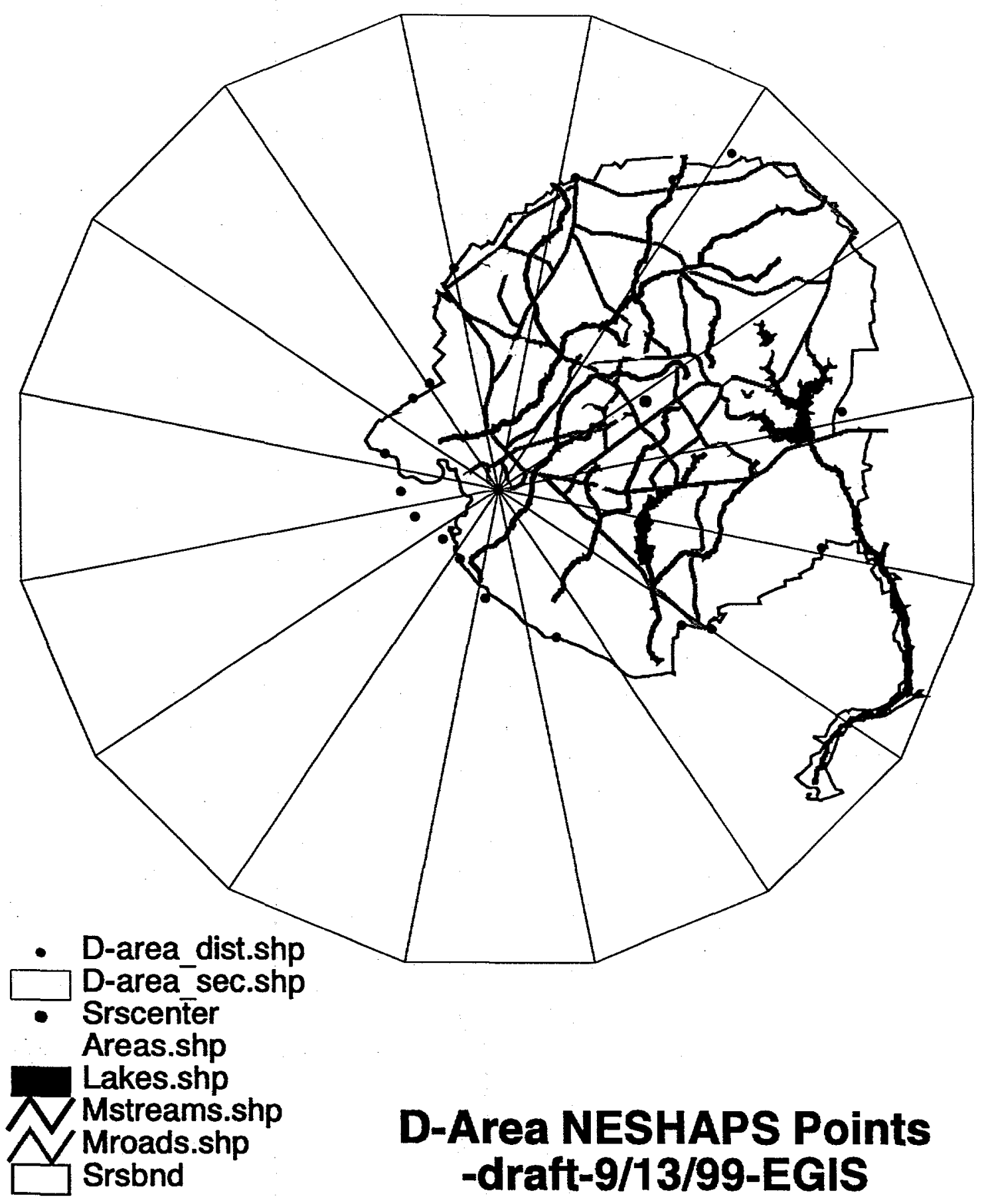




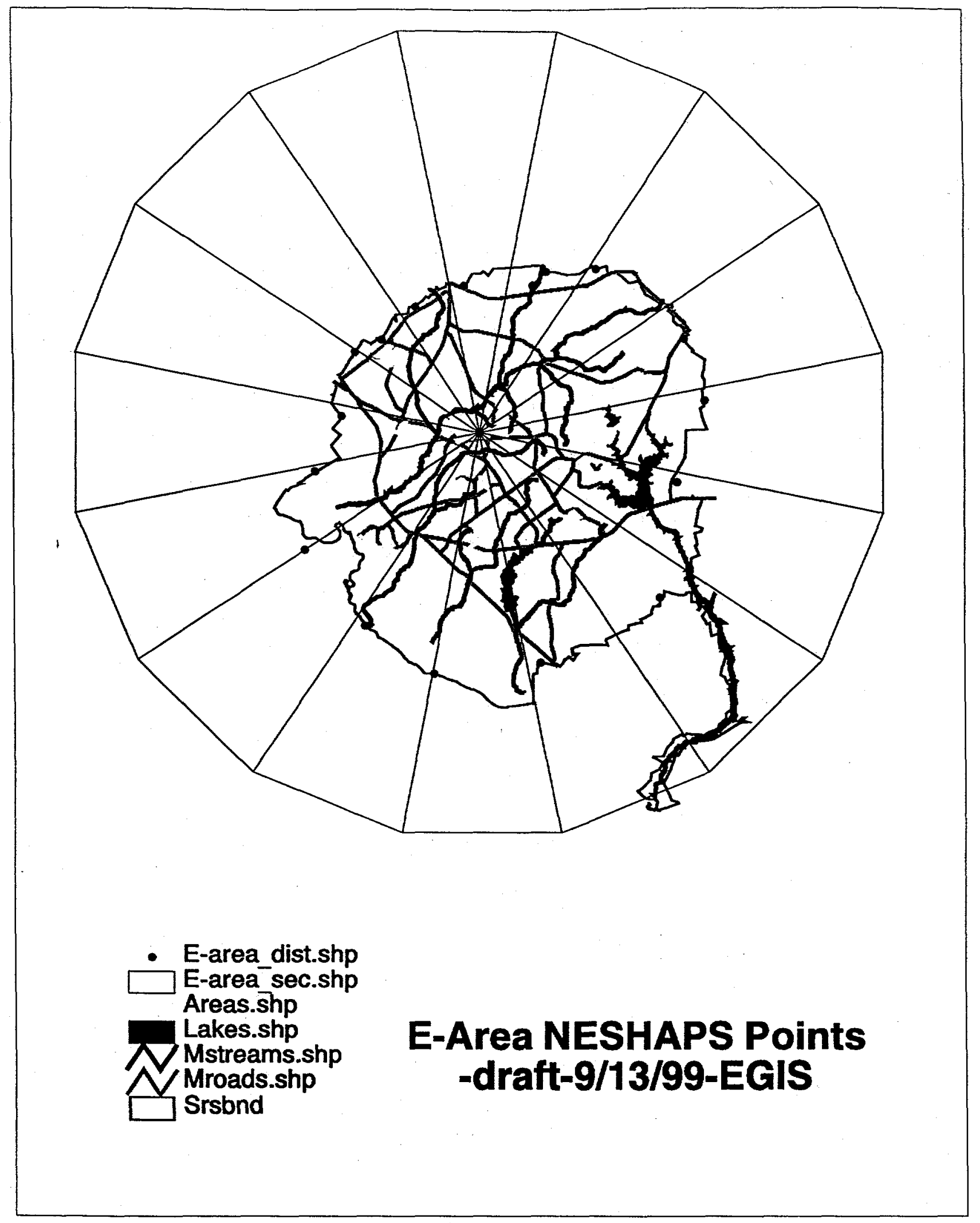




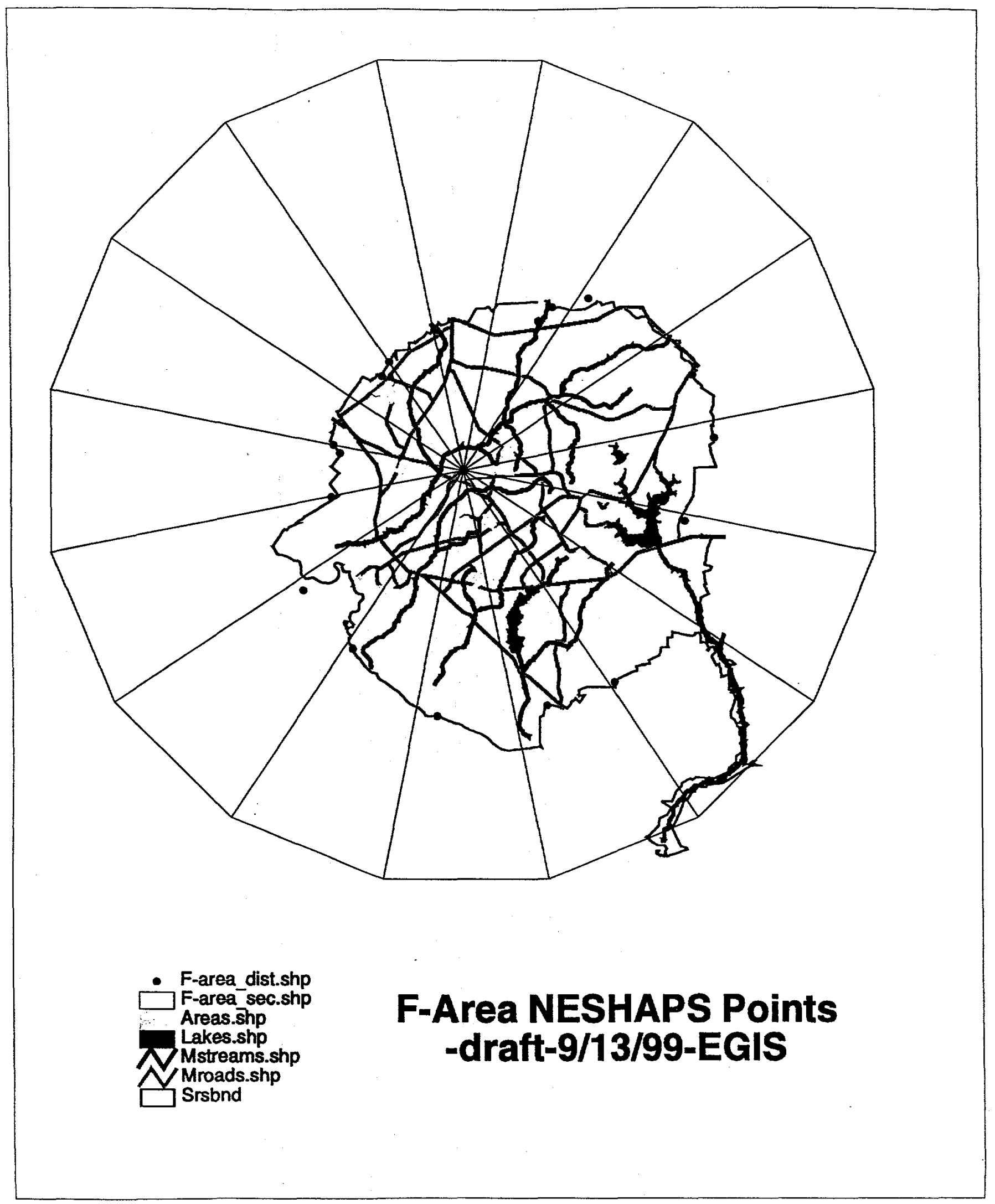




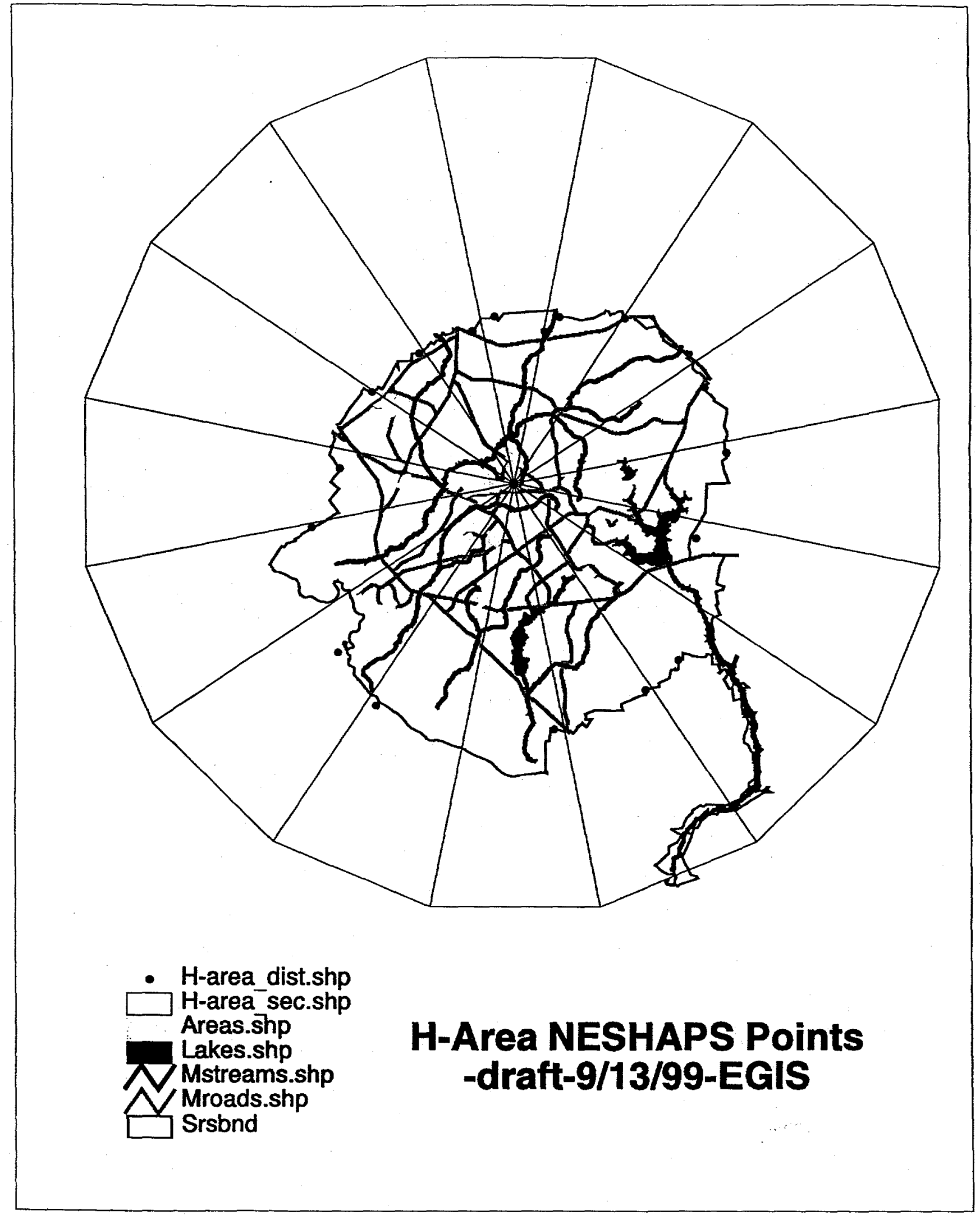




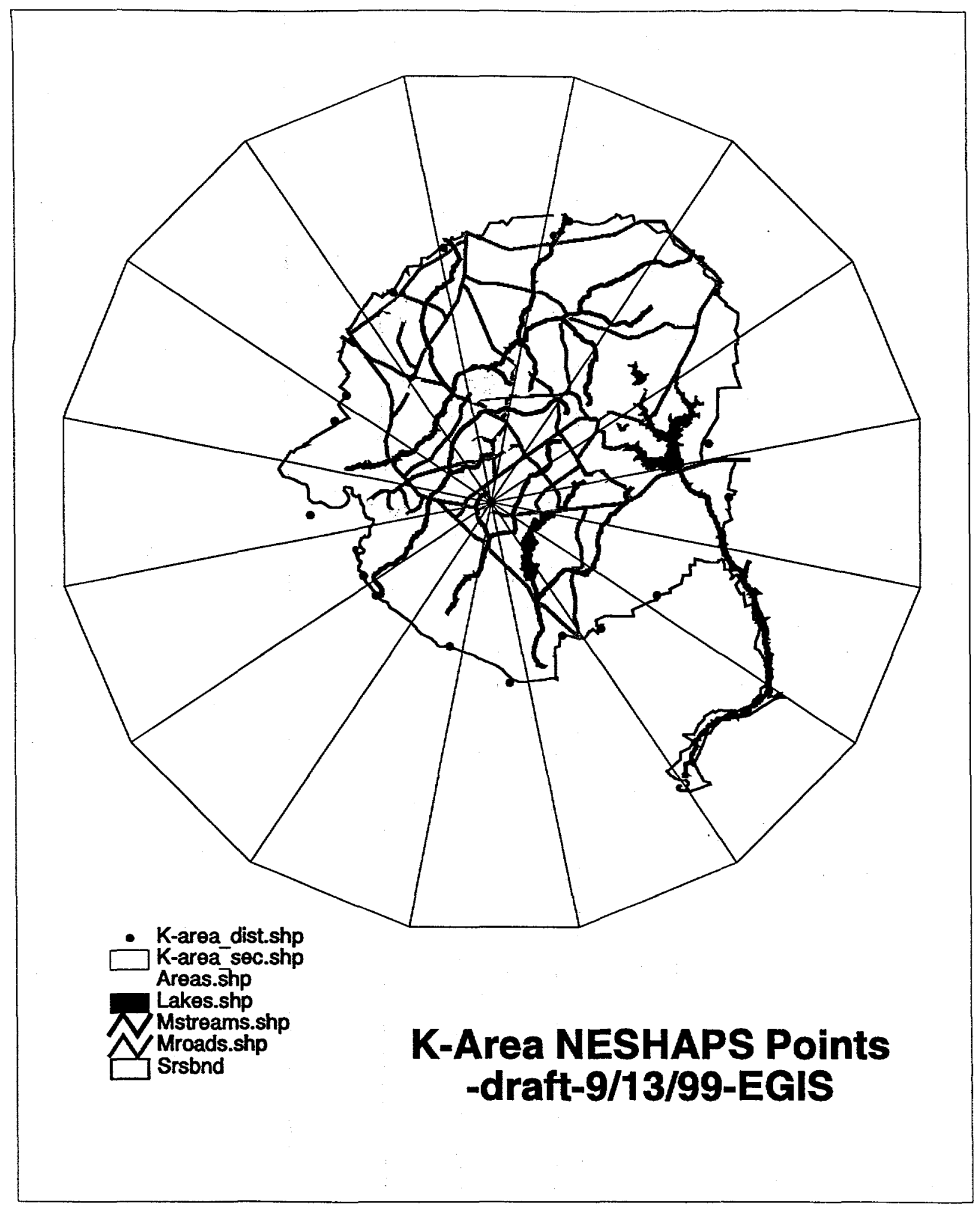




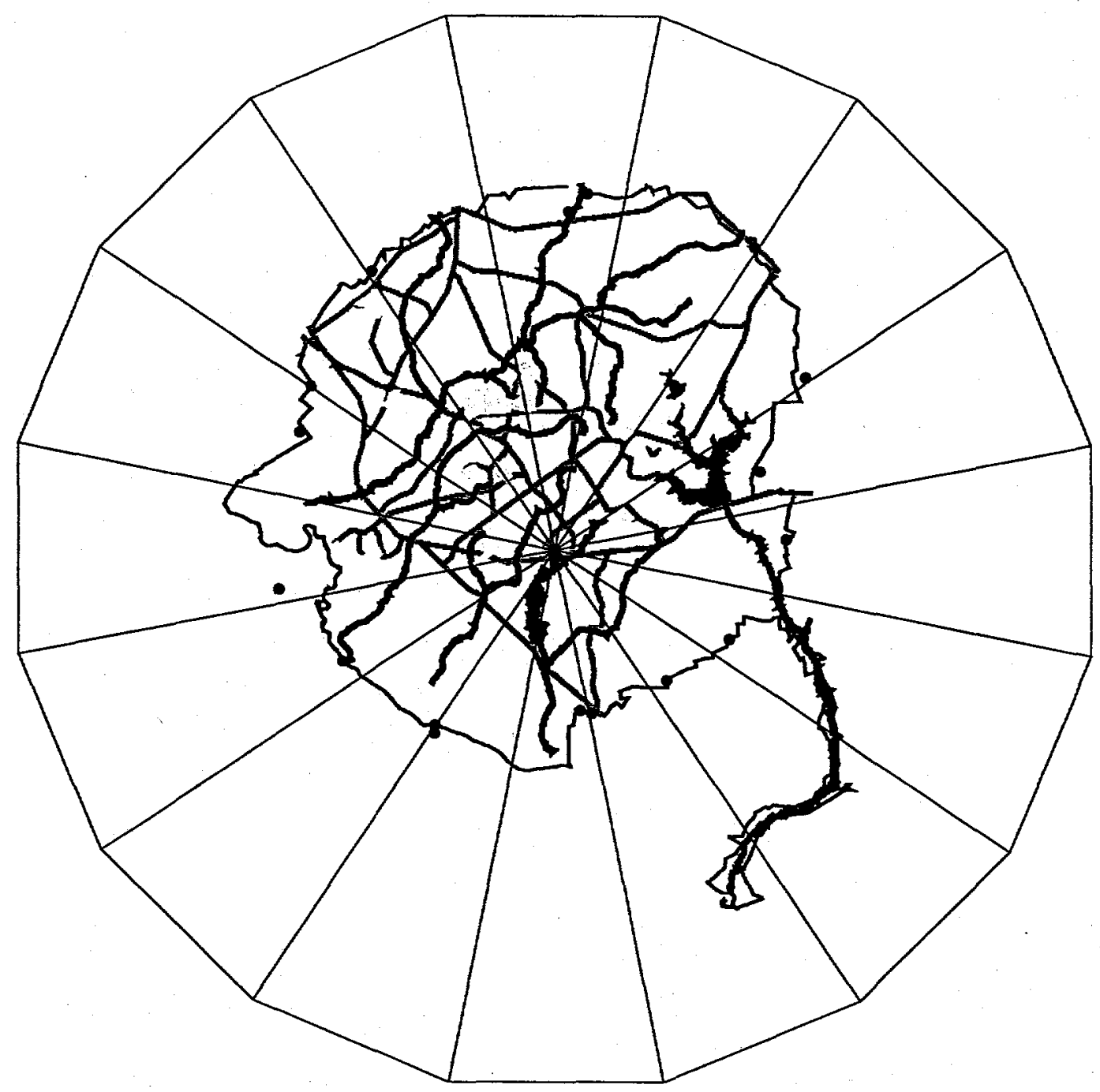

- L-area_dist.shp L-area_sec.shp Areas.shp Lakes.shp Mstreams.shp Mroads.shp Srsbnd

L-Area NESHAPS Points -draft-9/13/99-EGIS 


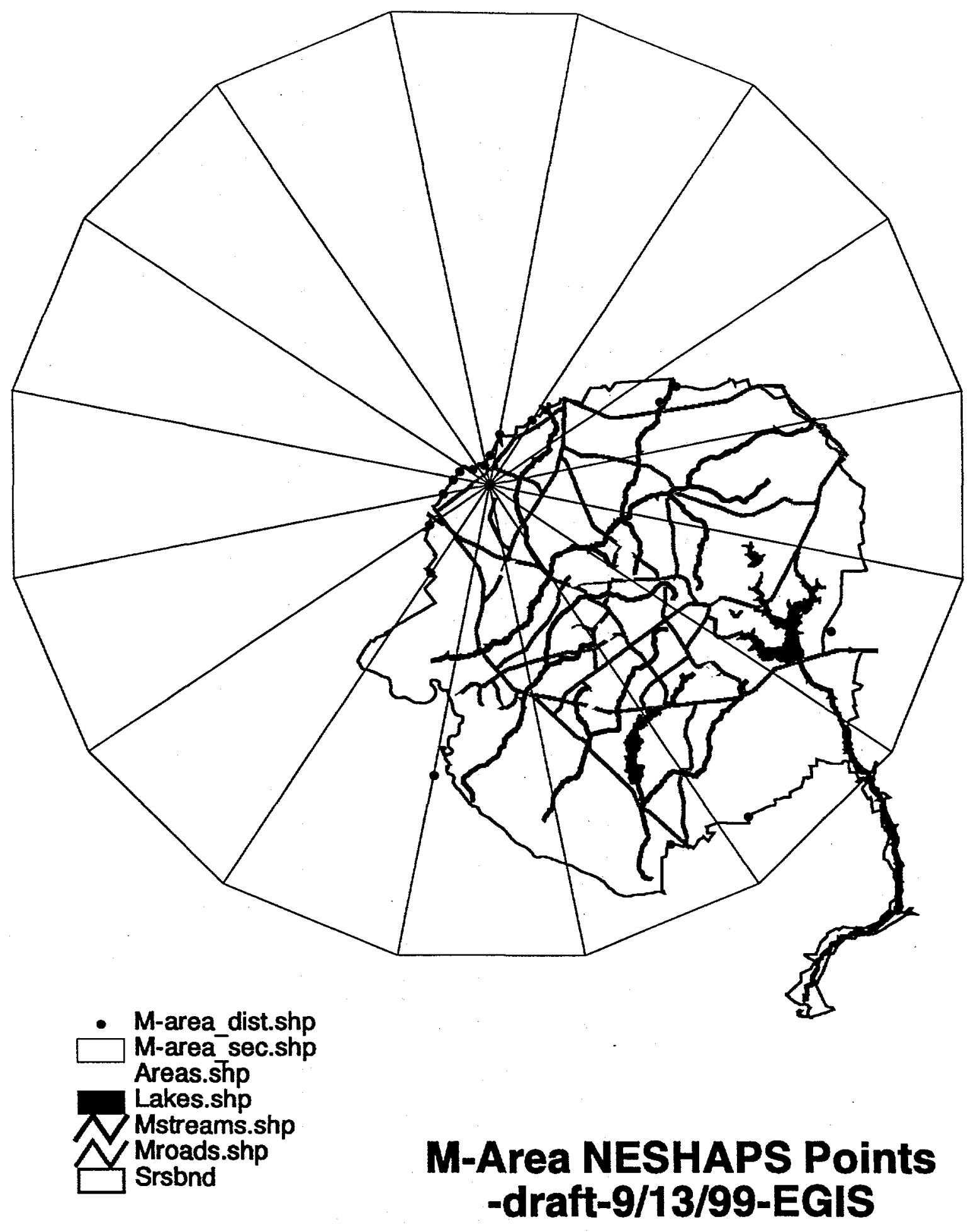




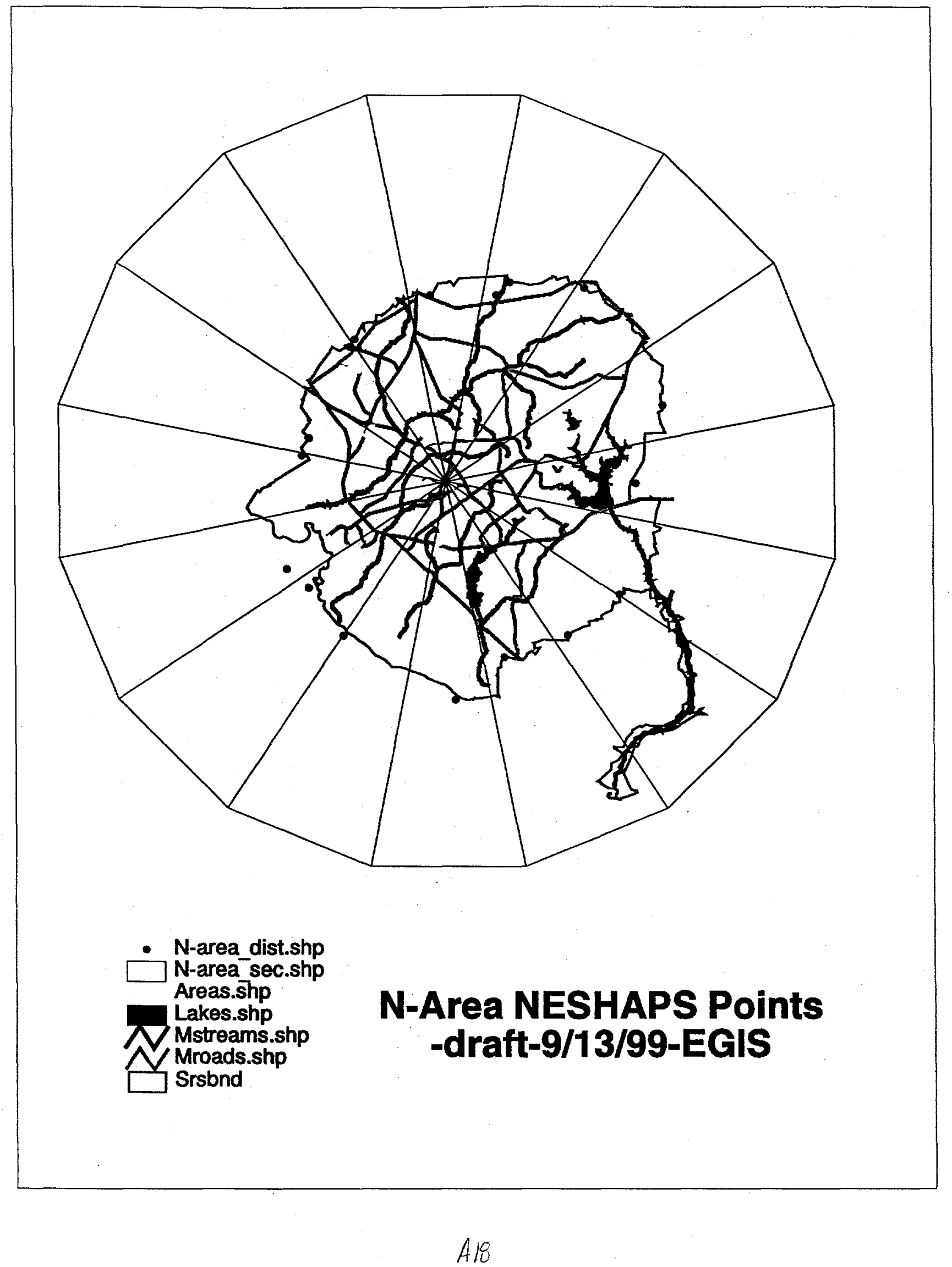




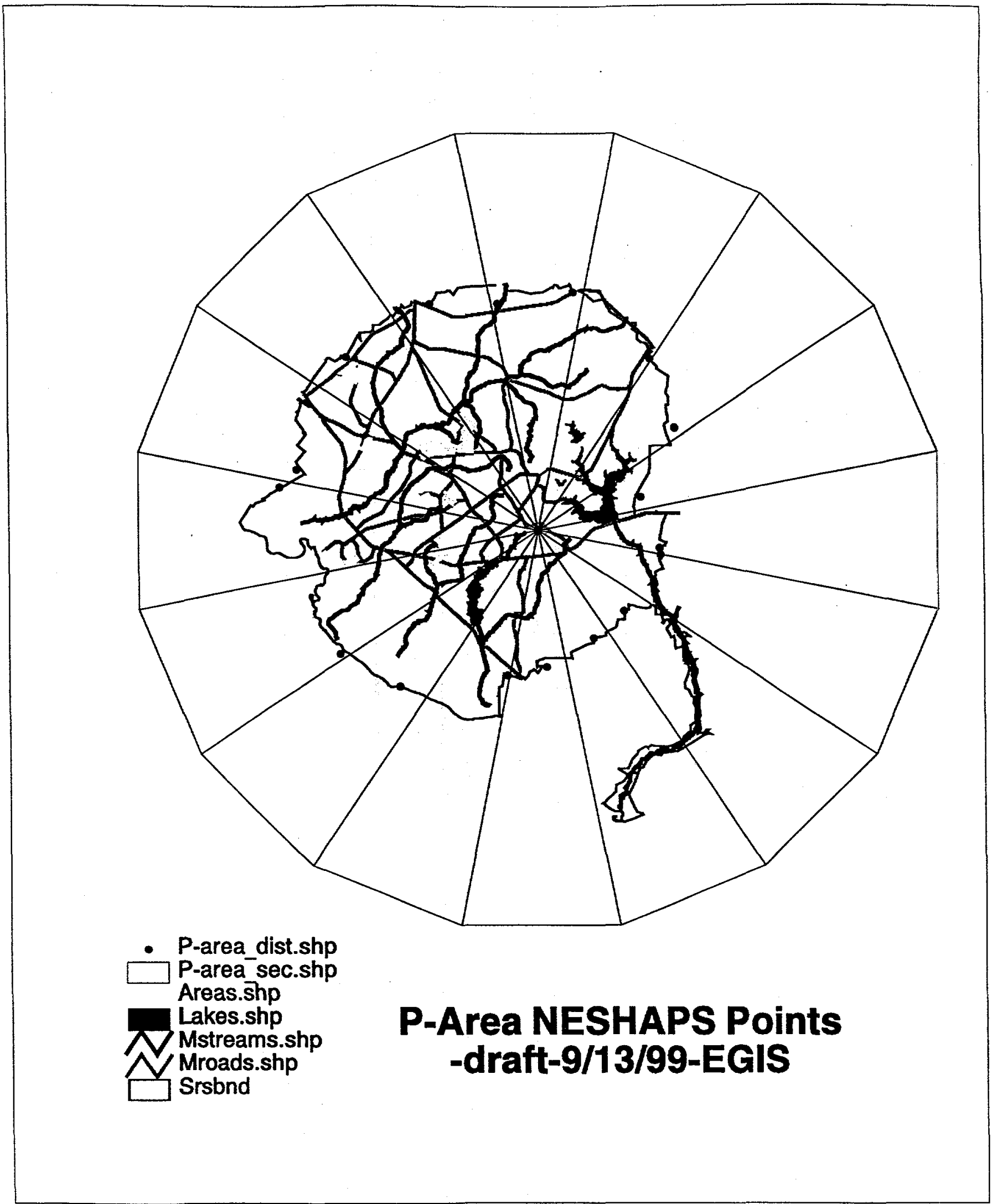




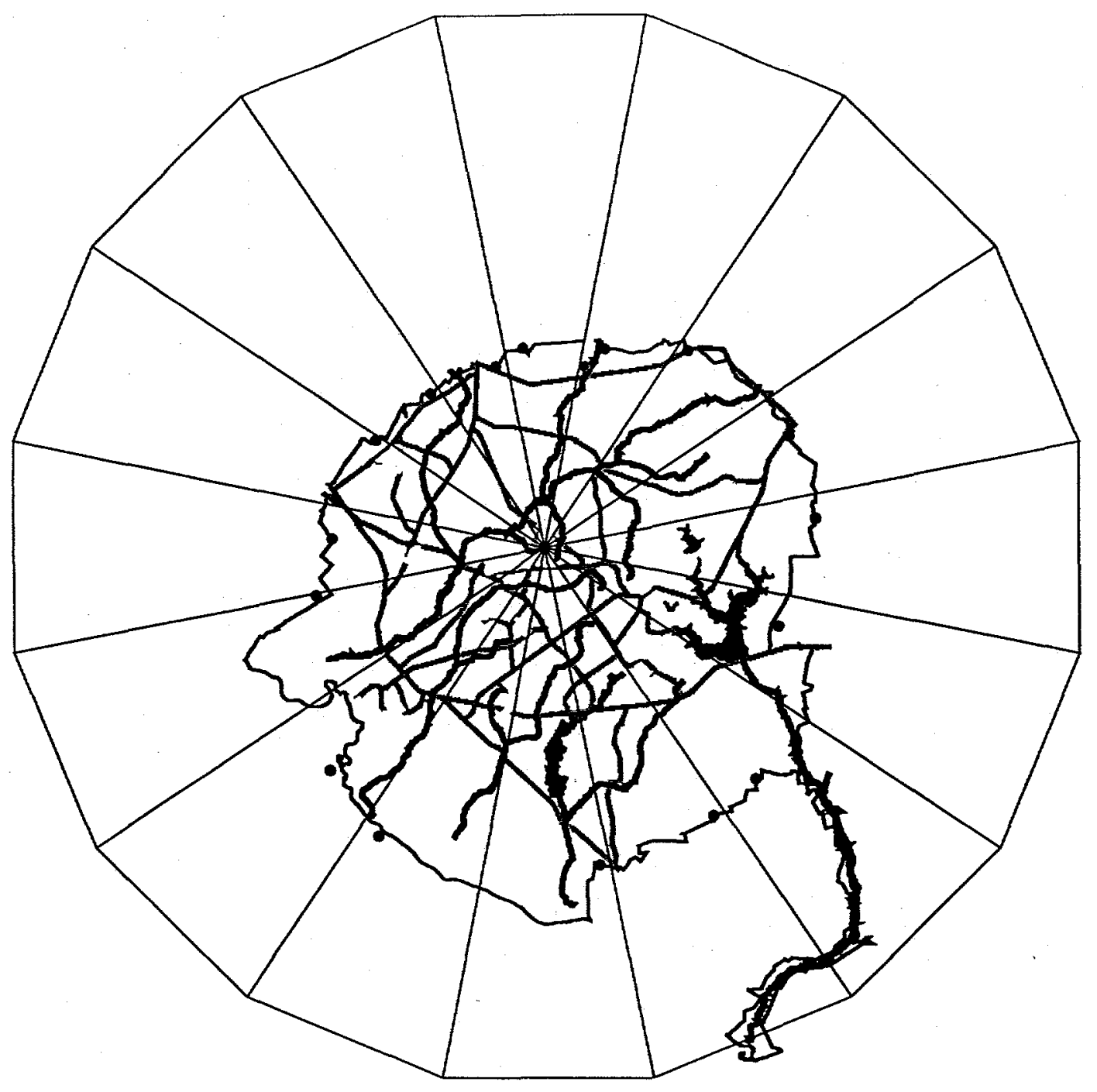

\section{- Sz-area_dist.shp}

Sz-area sec.shp

Areas.shp

Lakes.shp

Mstreams.shp

Mroads.shp

Srsbond

\section{SZ-Area NESHAPS Points -draft-9/13/99-EGIS}




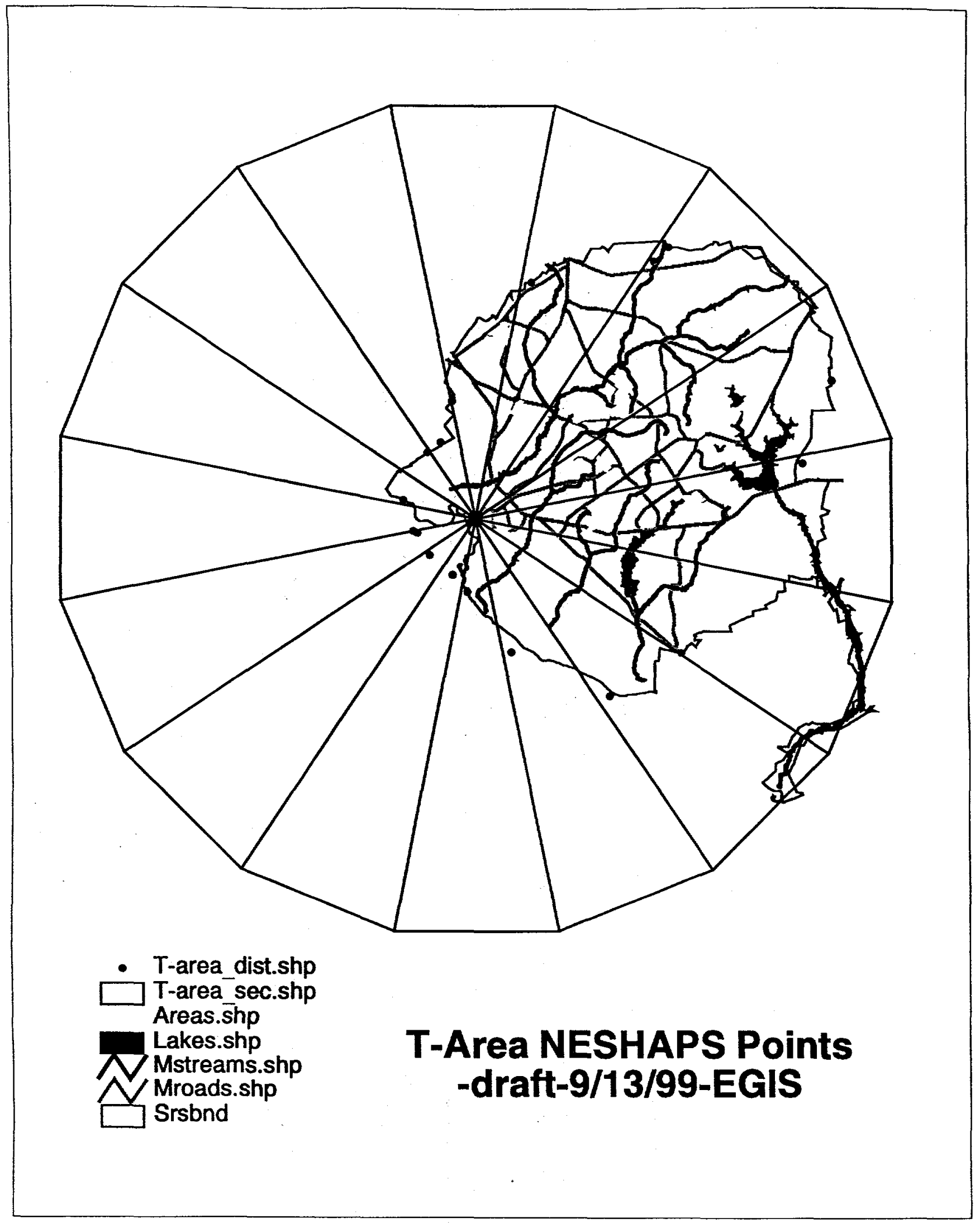


APPENDIX B. Offsite MEI Locations for 1990 
Table B1. Distance to Nearest Residence, School, Business, or Farm from 1990 Study (Bold shows worst sector)

\begin{tabular}{|c|c|c|c|c|c|c|c|c|c|c|c|c|c|c|}
\hline Sector & $\overline{\text { A-Area }}$ & $\overline{\mathrm{APT}}$ & C-Area & Center & D-Area & E-Area & F-Area & H-Area & K-Area & L-Area & M-Area & P-Area & S/Z-Area & -Area \\
\hline $\mathbf{N}$ & 1,960 & 9,350 & 15,540 & 16,100 & 15,080 & 11,050 & 11,310 & 12,370 & 19,460 & 20,970 & 1,980 & 19,010 & 10,970 & 12,340 \\
\hline NNE & 4,230 & 10,600 & 18,130 & 16,260 & 20,970 & 13,790 & 14,180 & 12,820 & 21,420 & 21,720 & 4,300 & 16,140 & 11,430 & 21,030 \\
\hline NE & 6,940 & 11,460 & 18,440 & 7,550 & 27,760 & 16,690 & 17,500 & 14,930 & 22,320 & 17,800 & 7,160 & 13,580 & 14,170 & 28,190 \\
\hline ENE & 14,190 & 12,370 & 17,980 & 16,580 & 22,930 & 17,830 & 18,700 & 15,540 & 16,140 & 12,670 & 14,480 & 8,450 & 15,240 & 25,290 \\
\hline E & 22,340 & 13,270 & 16,910 & 13,200 & 23,980 & 18,060 & 19,010 & 15,540 & 17,200 & 13,580 & 22,860 & 9,650 & 16,000 & 24,080 \\
\hline ESE & 24,300 & 12,970 & 18,060 & 15,300 & 17,050 & 16,080 & 17,040 & 13,730 & 13,730 & $1.1,610$ & 24,680 & 10,260 & 14,630 & 19,500 \\
\hline SE & 27,920 & 15,990 & 16,300 & 15,300 & 14,930 & 19,280 & 19,760 & 17,500 & 12,140 & 10,560 & 28,190 & 9,350 & 18,890 & 17,370 \\
\hline SSE & 26,710 & 19,310 & 15,540 & 15,300 & 11,920 & 18,890 & 19,010 & 17,650 & 11,010 & 9,960 & 26,820 & 9,500 & 19,500 & 11,120 \\
\hline $\mathbf{s}$ & 20,210 & 20,670 & 16,760 & 15,130 & 9,800 & 21,180 & 19,310 & 18,180 & 13,420 & 9,650 & 20,270 & 10,860 & 20,110 & 5,940 \\
\hline ssw & 7,240 & 23,230 & 15,240 & 17,230 & 5,730 & 17,140 & 16,290 & 20,270 & 11,540 & 14,030 & 7,160 & 11,770 & 21,640 & 6,550 \\
\hline sw & 5,430 & 22,020 & 13,100 & 16,420 & 7,390 & 16,380 & 15,230 & 18,340 & 11,920 & 13,430 & 5,330 & 17,500 & 19,660 & 5,030 \\
\hline WSW & 3,470 & 15,690 & 13,180 & 17,230 & 6,640 & 13,260 & 11,920 & 15,290 & 10,860 & 14,180 & 3,350 & 18,400 & 13,710 & 4,270 \\
\hline$w$ & 2,560 & 15,080 & 11,580 & 14,170 & 6,490 & 10,820 & 9,500 & 13,190 & 13,120 & 16,890 & 2,440 & 20,360 & 12,950 & 6,250 \\
\hline WNW & 2,260 & 12,520 & 10,210 & 13,850 & 8,450 & 11,430 & 10,110 & 12,520 & 12,820 & 16,140 & 2,130 & 19,160 & 10,970 & 5,940 \\
\hline NW & 1,660 & 10,260 & 12,340 & 14,650 & 8,300 & 10,360 & 9,350 & 12,070 & 13,120 & 18,550 & 1,520 & 20,060 & 10,970 & 6,400 \\
\hline NNW & 1,360 & 9,960 & 12,880 & 15,300 & 8,450 & 11,660 & 10,260 & 11,770 & 16,890 & 19,910 & 1,370 & 19,760 & 10,360 & 6,860 \\
\hline
\end{tabular}


WESTINGHOUSE SAVANNAH RIVER COMPANY

MAXIMALLY EXPOSED OFFSITE INDIVIDUAL LOCATION DETERMINATION FOR NESHAPS COMPLIANCE

\section{DISTRIBUTION (12)}

S. Wood, 773-A

D. B. Moore-Shedrow, 773-A

J.B. Gladden, 773-42A

G.T. Jannik, 773-42A

A. A. Simpkins, $773-42$ A

G. Whitney, 703-A

SRTC Records(4), 773-52A

ED Records(3), 773-42A 